\title{
Retinol-binding protein 7 is an endothelium-specific PPAR y cofactor mediating an antioxidant response through adiponectin
}

\author{
Chunyan Hu, ${ }^{1}$ Henry L. Keen, ${ }^{1}$ Ko-Ting Lu, ${ }^{1}$ Xuebo Liu, ${ }^{1}$ Jing Wu, ${ }^{1}$ Deborah R. Davis, ${ }^{1}$ \\ Stella-Rita C. Ibeawuchi, ${ }^{1}$ Silke Vogel, ${ }^{3}$ Frederick W. Quelle, ${ }^{1}$ and Curt D. Sigmund ${ }^{1,2}$ \\ 'Department of Pharmacology, ${ }^{2} \mathrm{UIHC}$ Center for Hypertension Research, Roy J. and Lucille A. Carver College of Medicine, \\ University of lowa, lowa City, lowa, USA. ${ }^{3}$ Duke-NUS Medical School, Singapore, Singapore.
}

Impaired PPAR $\gamma$ activity in endothelial cells causes oxidative stress and endothelial dysfunction which causes a predisposition to hypertension, but the identity of key PPAR $\gamma$ target genes that protect the endothelium remain unclear. Retinol-binding protein 7 (RBP7) is a PPAR $\gamma$ target gene that is essentially endothelium specific. Whereas RBP7-deficient mice exhibit normal endothelial function at baseline, they exhibit severe endothelial dysfunction in response to cardiovascular stressors, including high-fat diet and subpressor angiotensin II. Endothelial dysfunction was not due to differences in weight gain, impaired glucose homeostasis, or hepatosteatosis, but occurred through an oxidative stress-dependent mechanism which can be rescued by scavengers of superoxide. RNA sequencing revealed that RBP7 was required to mediate induction of a subset of PPAR $\gamma$ target genes by rosiglitazone in the endothelium including adiponectin. Adiponectin was selectively induced in the endothelium of control mice by high-fat diet and rosiglitazone, whereas RBP7 deficiency abolished this induction. Adiponectin inhibition caused endothelial dysfunction in control vessels, whereas adiponectin treatment of RBP7-deficient vessels improved endothelium-dependent relaxation and reduced oxidative stress. We conclude that RBP7 is required to mediate the protective effects of PPAR $\gamma$ in the endothelium through adiponectin, and RBP7 is an endothelium-specific PPAR $\gamma$ target and regulator of PPAR $\gamma$ activity.

Conflict of interest: The authors have declared that no conflict of interest exists.

Submitted: November 11, 2016 Accepted: February 8, 2017 Published: March 23, 2017

Reference information: JCI Insight. 2017;2(6):e91738. https:// doi.org/10.1172/jici.insight.91738.

\section{Introduction}

Cardiovascular disease is the leading cause of mortality worldwide. Endothelial dysfunction, characterized by impaired endothelium-dependent relaxation and reduced nitric oxide (NO) bioavailability, is a wellestablished cardiovascular risk factor (1). The nuclear receptor transcription factor peroxisome proliferator-activated receptor $\gamma$ (PPAR $\gamma$ ) is best known as a master regulator of adipocyte differentiation (2). Loss of PPAR $\gamma$ causes insulin resistance $(3,4)$, whereas its activation by thiazolidinediones improves insulin sensitivity (5). In addition to its ability to improve glucose metabolism, the PROactive trial revealed that pioglitazone lowered blood pressure (BP) and improved vascular function in type 2 diabetes (6). Consistent with a protective effect of PPAR $\gamma$ on BP, mutations in PPAR $\gamma$ cause early-onset severe hypertension that is refractory to antihypertensive treatment $(7,8)$. There is now convincing evidence that PPAR $\gamma$ and its downstream signaling pathways play a critical role in the regulation of BP and vascular function through their actions in both vascular smooth muscle (9-11) and endothelium (12-14). In endothelial cells, PPAR $\gamma$ exerts protective effects by regulating expression of genes related to oxidative stress and inflammation, regulating production of NO, and maintaining the balance of vascular constrictors and dilators, which influence the overlying vascular smooth muscle (15-19). However, the full range of molecular targets of PPAR $\gamma$ and the protective mechanisms they exert in the endothelium remains to be fully elucidated.

Retinol-binding protein 7 (RBP7) is a PPAR $\gamma$ target gene that is robustly induced by PPAR ligands, repressed by dominant-negative PPAR $\gamma$, and contains bona fide PPAR $\gamma / R X R$ binding sites in the genome (20-23). RBP7 is an intracellular RBP belonging to the family of intracellular lipid-and fatty acid-binding proteins (FABPs) (24). Early studies demonstrated that RBP7 (then known as CRBPIII) is involved in lipid 
and whole-body energy metabolism (20). However, recent evidence suggests that RBP7 is an endotheliumenriched transcript (25), and other data suggest that RBP7 may be exclusively expressed in endothelium, particularly in small blood vessels (26). The fact that RBP7 is an endothelium-specific PPAR $\gamma$ target with an unknown physiological function in the endothelium makes it a rational and interesting target for study.

Adiponectin (AdipoQ) is a PPAR $\gamma$ target that is well recognized as a protective adipokine (27). AdipoQ is secreted from adipose tissue and exerts protective effects on the cardiovascular system. High plasma levels of AdipoQ are associated with lower risk of cardiovascular disease (28), whereas hypoadiponectinemia causes chronic inflammation (29). Interestingly, reduced AdipoQ results in impaired endotheliumdependent relaxation (30), whereas AdipoQ administration increases NO bioavailability and reduces aortic oxidative stress $(31,32)$. In addition to adipose tissue, AdipoQ is expressed in the endothelium but its functional relevance remains unclear (33).

Based on RNA sequencing (RNA-Seq) data showing that Adipo $Q$ is induced by PPAR $\gamma$ agonist in carotid artery devoid of perivascular adipose tissue, we tested the hypothesis that both RBP7 and AdipoQ play a critical role in mediating vascular protection by endothelial PPAR $\gamma$. We demonstrate that RBP7-deficient mice exhibit normal endothelial function under baseline conditions, but exhibit endothelial dysfunction via an oxidative stress-dependent mechanism in response to either high-fat diet (HFD)induced obesity or a dose of angiotensin II (Ang-II) that does not cause hypertension in normal mice. Further, we demonstrate that RBP7 is required for the induction of many PPAR $\gamma$ target genes, including Adipo $Q$ by PPAR $\gamma$ in endothelium. Finally, we show that a neutralizing antibody against AdipoQ can induce endothelial dysfunction in vessels from Ang-II-treated C57BL/6J mice, and supplementation of AdipoQ can restore endothelial function in response to Ang-II in RBP7-deficient mice. These data define a previously unknown PPAR $\gamma / \mathrm{RBP} 7 /$ AdipoQ protective pathway in the endothelium, which when impaired results in oxidative stress and endothelial dysfunction.

\section{Results}

RBP7 expression is endothelium specific. Previous evidence using mice expressing LacZ inserted into the RBP7 locus suggested that RBP7 is selectively expressed in endothelium (26). We validated the endothelial specificity of $R B P 7$ expression. Endothelial cells and non-endothelial cells were enriched from control and RBP7-deficient mouse kidney, lung, and aorta using magnet-activated cell sorting (MACS). The fidelity of the MACS-based cell sorting was confirmed by the presence of Cd31 and Nos3 (encoding endothelial NOS) mRNA in the endothelial fractions but not in the non-endothelial fractions (Supplemental Figure 1; supplemental material available online with this article; https://doi.org/10.1172/jci.insight.91738DS1). The expression of these characteristic genes exclusively in the positively enriched cell fractions provides evidence that these represent endothelial cells. $R B P 7$ mRNA was easily detectable in the endothelial fractions from all 3 tissues (Figure 1A). However, the level of $R B P 7$ expression in non-endothelial cells from kidney, lung, and aorta was $12.1 \%, 10.2 \%$, and $4.8 \%$ of the level in endothelial cells, respectively. There was no $R B P 7$ mRNA detected in tissues from RBP7-deficient mice.

$R B P 7$ mRNA expression was induced by the PPAR $\gamma$ agonist rosiglitazone, and its induction was blunted by pretreatment with the PPAR $\gamma$ antagonist GW9662 in immortalized mouse lung endothelial cells (MLECs), which endogenously express RBP7 (Figure 1B). Similarly, the level of RBP7 protein in MLECs was induced by either rosiglitazone or PPAR $\gamma$ overexpression, and its expression was further increased by rosiglitazone when PPAR $\gamma$ was overexpressed (Figure 1, C and D). These data demonstrate that expression of RBP7 is endothelium specific or virtually so, and that RBP7 is a PPAR $\gamma$ target in endothelium. Importantly, given the endothelial specificity of RBP7 expression established by LacZ knockin (26) and the MACS enrichment, RBP7-deficient mice can be considered an endothelial cell-specific RBP7-deficient model.

$R B P 7$ deficiency causes endothelial dysfunction after HFD. Experiments were conducted to evaluate the functional importance of RBP7 in endothelium-dependent vascular function. At baseline, there was no difference in relaxation of the basilar artery (a cerebral artery) in response to the endothelium-dependent vasodilator acetylcholine (ACh, Figure 2A), nor the endothelium-independent vasodilator sodium nitroprusside (SNP, Figure 2B). However, in response to 8 weeks of HFD feeding, ACh-dependent vasodilation was selectively impaired in RBP7-deficient mice but not littermate controls (Figure 2A). There was no change in BP or heart rate as measured by radiotelemetry in RBP7-deficient or littermate controls fed either normal diet (ND) or HFD for 8 weeks (Figure 2C, and Supplemental Figure 2). 
A

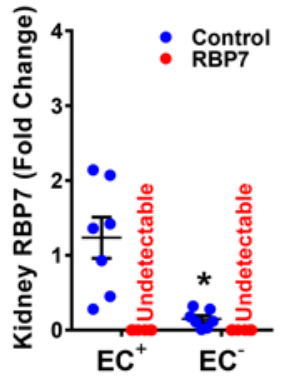

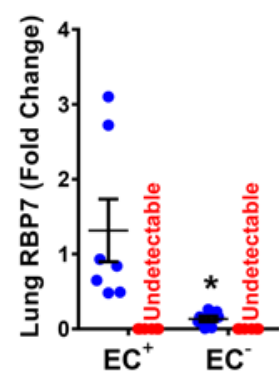

C

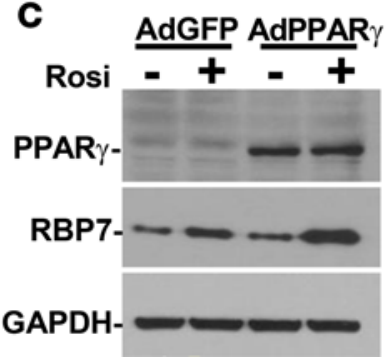

B

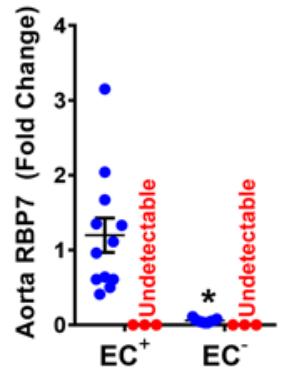

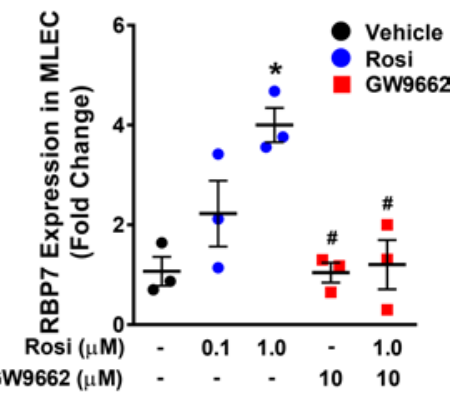

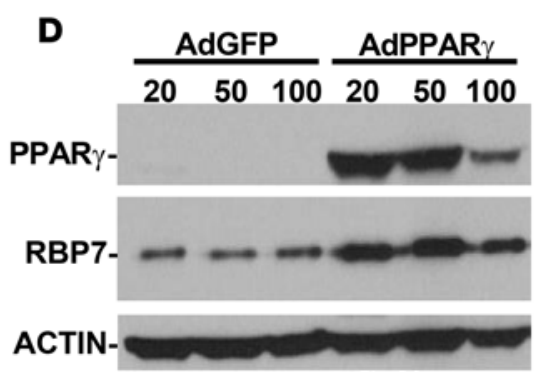

Figure 1. Endothelial specificity of retinol-binding protein 7 (RBP7). (A) Endothelial (EC+) and non-endothelial (EC-) cells were magnetically sorted from dissociated single cells isolated from kidney, lung, and aorta from control and RBP7deficient mice. RBP7 expression was measured by quantitative real-time RT-PCR. ${ }^{*} P<0.05 \mathrm{EC}^{+}$vs. EC ${ }^{-}$cells by Student's $t$ test. $n=6-7$ per group in kidney and lung, and 3-12 in aorta. (B) Expression of RBP7 mRNA was measured in mouse lung endothelial cells (MLECs) in response to 24 hours of the indicated dose of rosiglitazone (Rosi) or vehicle (DMSO) in the absence or presence of GW9662 $(10 \mu \mathrm{M})$. ${ }^{*} P<0.05$ Rosi $(1.0 \mu \mathrm{M})$ vs. DMSO; ${ }^{\#} P<0.05 \mathrm{GW} 9662$ vs. Rosi $(1.0 \mu \mathrm{M})$ by 1 -way ANOVA; $n=3$ per group. (C) The level of RBP7 protein was measured by Western blot from MLECs treated with vehicle or Rosi $(10 \mu \mathrm{M})$ for 24 hours that were infected with either adenovirus expressing GFP (AdGFP) or PPAR (AdPPAR $\gamma$ ) (see supplemental methods). Representative of 3 independent experiments. (D) The level of RBP7 protein was measured by Western blot from MLECs infected for 72 hours with either AdGFP or AdPPAR $\gamma$ at the indicated multiplicity of infection. The decrease in PPAR $\gamma$ expression in the highest dose is due to cell death. Representative of 4 independent experiments.

These data indicate that impaired endothelium-dependent relaxation observed in HFD-fed RBP7-deficient mice is not a result of increased arterial pressure.

Because preliminary studies revealed that 8 weeks of HFD feeding is insufficient to induce endothelial dysfunction in the carotid artery, a separate cohort of mice were fed HFD for 20 weeks. A similar pattern of HFD-induced endothelial dysfunction was observed in carotid artery from RBP7-deficient but not littermate control mice fed HFD for 20 weeks (Figure 2, D and E). As above, 20 weeks of HFD had no effect on the vasodilator responses to SNP (Figure 2E) or papaverine (data not shown), suggesting that the vascular smooth muscle was not impaired. Although BP was significantly elevated in 20-week HFD-fed compared with ND-fed mice (e.g., obesity-induced hypertension), no significant differences were found when comparing HFD-fed RBP7-deficient with HFD-fed control mice (Figure 2F and Supplemental Figure 2).

It is notable that the selective impairment in endothelial function observed in HFD-fed RBP7-deficient mice phenocopies the selective impairment in endothelial function observed in mice expressing dominantnegative mutations in PPAR $\gamma$ specifically in the endothelium (12). This suggests that the beneficial effects of PPAR $\gamma$ on endothelial function may be mediated by RBP7.

Endothelial dysfunction in RBP7-deficient mice is not caused by metabolic impairment. RBP7 was previously reported to regulate lipid metabolism (20). We therefore assessed if changes in systemic metabolism could explain the endothelial dysfunction observed in RBP7-deficient mice. There was no difference in body weight in RBP7-deficient and control mice fed ND until 24 weeks of age $(29.1 \pm 3.4 \mathrm{~g}$ control vs. $31.9 \pm 2.9$ g RBP7-deficient, $n=6$ ). Body weight gain was similar in both groups of mice after 8 weeks of HFD (Figure $3 \mathrm{~A}$ ), and total body weight was equal after 20 weeks of HFD (Figure 3B). Fasting glucose was highly variable and significantly increased in both HFD-fed groups, but there was no difference between RBP7deficient and control mice (Figure 3C). Similarly, HFD-fed RBP7-deficient and control mice exhibited the same level of glucose intolerance (Figure 3D), insulin insensitivity (Figure 3E), and lipid accumulation in 

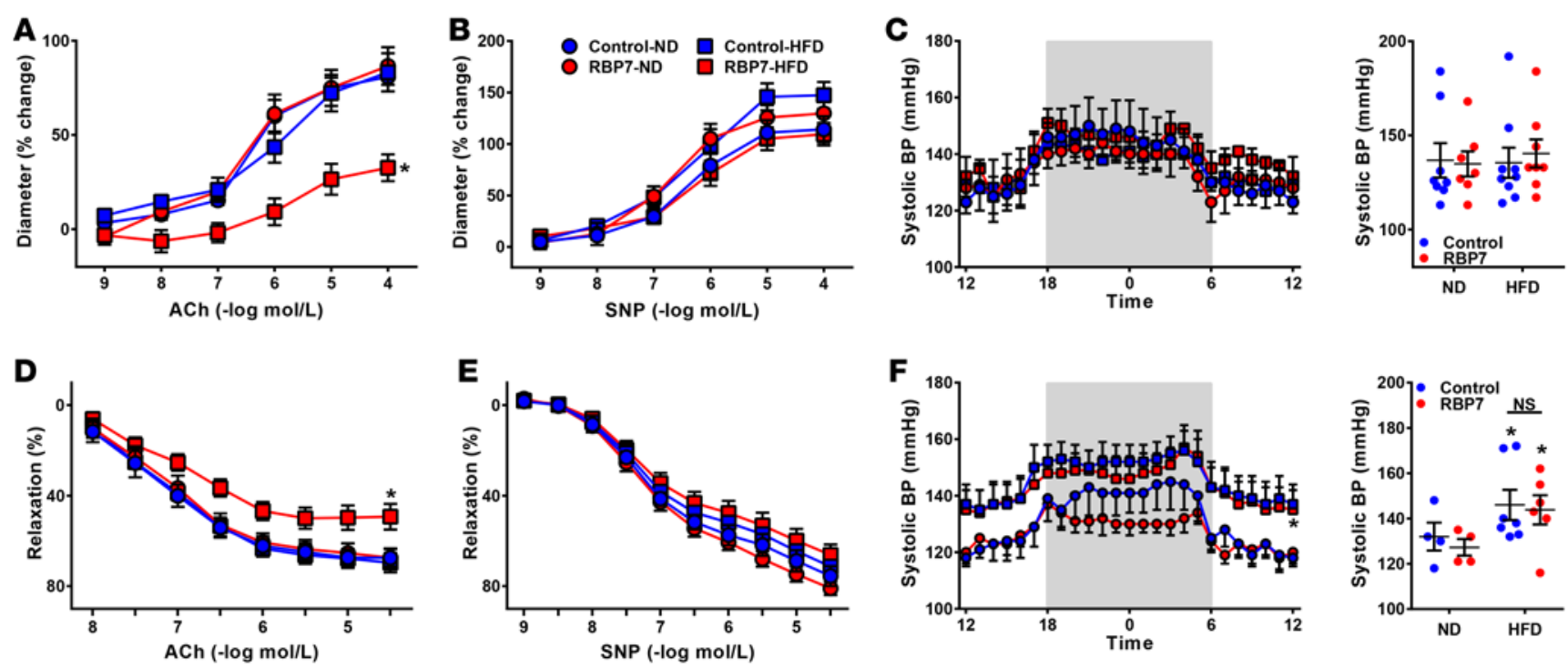

Figure 2. Arterial function in RBP7-deficient mice. (A and B) Vasodilation of the basilar artery from control and retinol-binding protein 7-deficient (RBP7-deficient) mice fed either normal diet (ND) or high-fat diet (HFD) for 8 weeks was measured in response to acetylcholine (ACh, A) and sodium nitroprusside (SNP, B). ${ }^{*} P<0.05$ vs. all other curves by 2-way repeated measures (RM) ANOVA; $n=7-9$ per group. (C) Systolic BP was measured by radiotelemetry in RBP7-deficient and control mice after 8 weeks of ND or HFD ( $n=7-9$ per group). (D and E) Vasodilation of the carotid artery from control and RBP7-deficient mice fed either ND or HFD for 20 weeks was measured in response to ACh (D) and SNP (E). ${ }^{*} P<0.05$ vs. all other curves by 2 -way RM ANOVA; $n=7$ per group. (F) Systolic BP was measured by radiotelemetry in RBP7-deficient and control mice after 20 weeks of ND or HFD ( $n=4-7$ per group). ${ }^{*} P<0.05$ HFD vs. ND by genotype by 2 -way and 3-way ANOVA. All data are the mean $\pm \mathrm{SEM}$. NS, nonsignificant.

liver (Figure 3F). The level of plasma RBP4, a circulating RBP which has been reported to convey cardiometabolic risk (34), did not differ significantly between HFD- and ND-fed mice (Figure 3G). These results suggest that the endothelial dysfunction selectively observed in HFD-fed RBP7-deficient mice is unlikely a result of systemic metabolic dysfunction because control mice fed HFD exhibited the same level of metabolic dysfunction but did not exhibit endothelial dysfunction.

Oxidative stress causes endothelial dysfunction in HFD-fed RBP7-deficient mice. Oxidative stress is a major contributor to endothelial dysfunction (1). Thus, experiments were conducted to evaluate whether a similar mechanism is operant in RBP7-deficient mice. Based on dihydroethidium staining, superoxide production was significantly increased in carotid arteries from HFD-fed RBP7-deficient mice (Figure 4A). We independently confirmed the presence of oxidative stress in the carotid artery of HFD-fed RBP7-deficient mice by nitrotyrosine immunofluorescence (Figure $4 \mathrm{~B}$ and Supplemental Figure 3). Nitrotyrosine staining was observed in both endothelium (see arrows) and throughout the media. This localization of nitrotyrosine staining is consistent with oxidative stress-induced endothelial dysfunction in other model systems $(35,36)$.

We next evaluated if attenuation of superoxide would ameliorate endothelial dysfunction. Basilar artery was incubated with the superoxide scavenger Tempol $(1 \mathrm{mmol} / \mathrm{l})$ or polyethylene-glycolated superoxide dismutase (PEG-SOD, $100 \mathrm{U} / \mathrm{ml}$ ) for 30 minutes. Endothelium-dependent ACh-induced relaxation was restored to normal in basilar artery from HFD-fed RBP7-deficient mice after incubation with either antioxidant (Figure 4, C and D). We also asked if the improved vasodilation by SOD was due to accumulation of $\mathrm{H}_{2} \mathrm{O}_{2}$, a potent vasodilator. Preincubation of the vessel with polyethylene-glycolated catalase (PEG-CAT) along with PEG-SOD did not impair the vasodilation response to ACh (Figure 4E). That PEG-CAT prevented the vasodilation of basilar artery from normal mice by $\mathrm{H}_{2} \mathrm{O}_{2}$ showed it was functioning appropriately in our assay (Figure 4F). These data suggest that the improvement in vasodilatation was due to a decrease in superoxide, not an accumulation of $\mathrm{H}_{2} \mathrm{O}_{2}$.

Consistent with these functional studies, expression of the pro-oxidant genes Nox2 and Nox4 were significantly increased in HFD-fed RBP7-deficient mice (Supplemental Figure 4). In contrast, expression of the antioxidant Cat gene was significantly decreased in HFD-fed RBP7-deficient mice. There was no significant change in expression of Sod1, Sod2, Sod3, or Nos3. These results indicate that oxidative stress is mechanistically involved in the endothelial dysfunction in HFD-fed RBP7-deficient mice. 


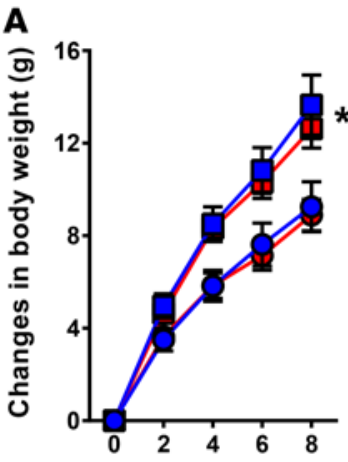

Time After Diet Started (weeks)

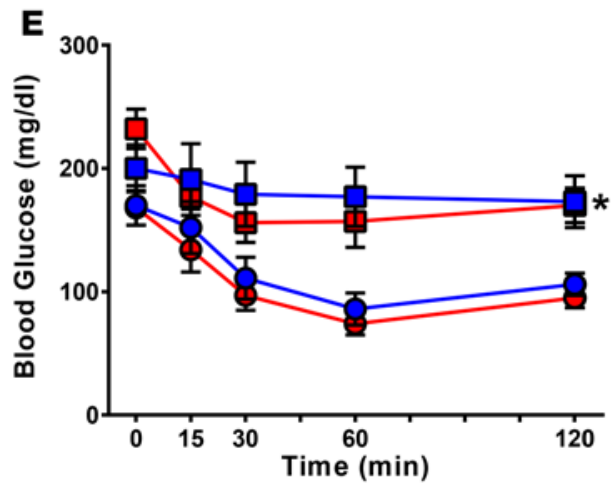

B

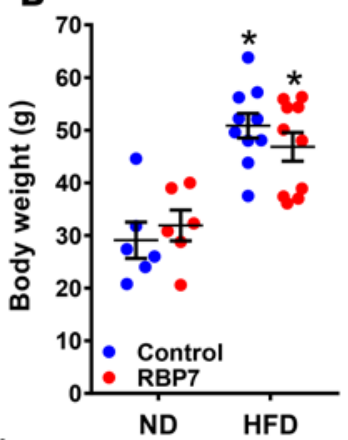

C

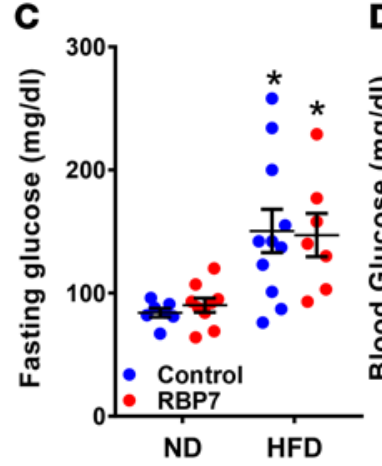

D

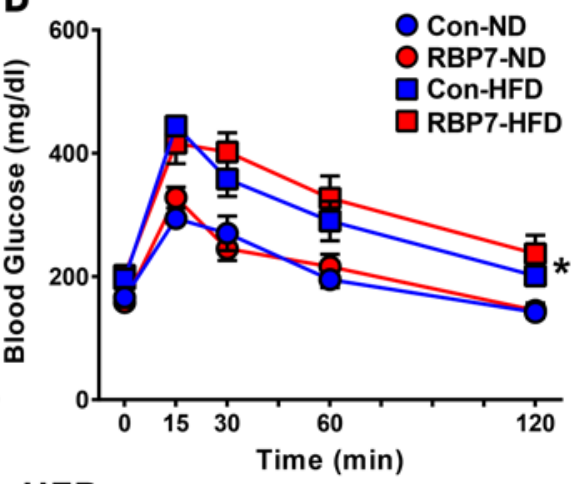

ND

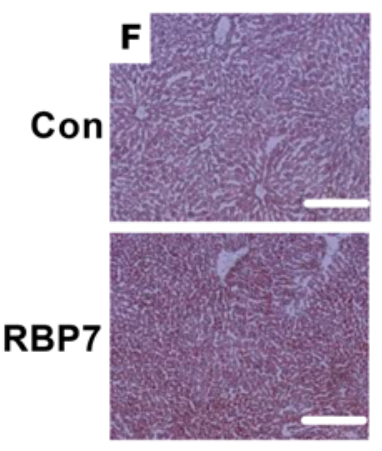

HFD

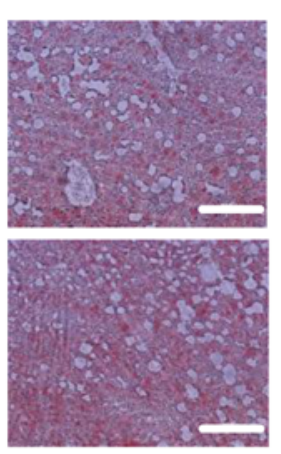

Figure 3. Metabolic parameters. (A) Changes in body weight from 0 to 8 weeks in ND-fed and HFD-fed control and RBP7-deficient mice were recorded. ${ }^{*} P<0.05$ HFD vs. ND by genotype by 2 -way repeated measures (RM) ANOVA; $n=11-18$ per group. (B-E) Body weight (B, $n=6-10$ per group), fasting glucose (C, $n=7-11$ per group), glucose tolerance test ( $\mathbf{D}, n=6-7$ per group), insulin tolerance test (E, $n=6-7$ per group) at 20 weeks in ND-fed and HFD-fed control and RBP7-deficient mice. ${ }^{*} P<0.05$ HFD vs. ND by genotype by 2 -way ANOVA in $\mathbf{B}$ and $\mathbf{C}$ and by 2 -way RM ANOVA in $\mathbf{D}$ and $\mathbf{E}$. (F) Oil red 0 staining of liver sections at 20 weeks in ND-fed and HFD-fed control and RBP7-deficient mice. Scale bars: $200 \mu \mathrm{m}$. (G) Plasma RBP4 measured at 20 weeks in ND-fed and HFD-fed control and RBP7-deficient mice. $n=7-9$ per group by 2-way ANOVA. All data are the mean \pm SEM.

Con, control; HFD, high-fat diet; ND, normal diet; RBP7, retinol-binding protein 7.

RBP7 is protective in Ang-II-infused mice. To examine the importance of RBP7 in response to another cardiovascular stressor, Ang-II was administered to RBP7-deficient and control mice. First, systolic BP was measured by tail cuff in a separate large cohort of control and RBP7-deficient mice to ensure that we used a population of mice that were phenotypically similar to those employed in the initial studies where BP was measured by radiotelemetry. Although systolic BP varied among mice, there was no significant difference in the control or RBP7-deficient groups (Figure 5A). We next tested a dose of Ang-II (termed a pressor dose) which markedly increases BP quickly. Systolic BP was increased similarly in both RBP7-deficient and control mice after 2 weeks of pressor Ang-II infusion (Supplemental Figure 5A). The relaxation response to ACh was equally impaired in carotid artery from pressor Ang-II-treated RBP7-deficient and control mice (Supplemental Figure 5B), whereas there was a modest rightward shift in SNP response for the AngII-infused RBP7-deficient mice compared with vehicle-treated control mice (Supplemental Figure 5C).

To exclude the potential confounding influence of increased BP on vascular function, experiments were conducted in mice infused with a low dose (termed a subpressor dose) of Ang-II, which does not elevate BP in normal mice. Systolic BP was measured in a subgroup of this cohort. There was no significant change in systolic BP in response to subpressor Ang-II in either group of mice (Figure 5B). Interestingly however, ACh-induced relaxation was selectively impaired in subpressor Ang-II-infused RBP7-deficient mice compared with other groups (Figure 5C). There was no difference in SNP-induced vasodilation (Figure 5D). Like the selective effect of HFD, superoxide (Figure 5E) and nitrotyrosine (Figure 5F and Supplemental Figure 6) levels were significantly and selectively increased in carotid artery from subpressor Ang-II-infused RBP7-deficient mice. Tempol incubation ameliorated the impairment in ACh-induced relaxation (Figure $5 \mathrm{G}$ ). In total, these data suggest that RBP7 provides protection against oxidative stress in response to 2 different cardiovascular risk factors, HFD and Ang-II. 
A

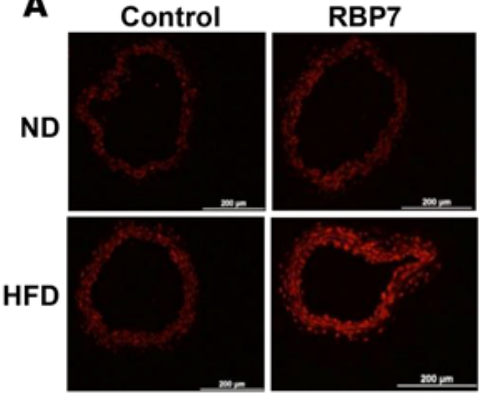

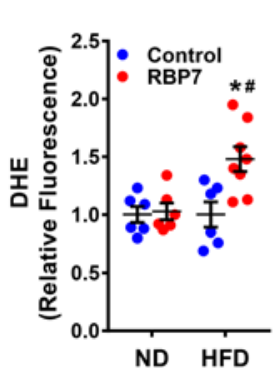

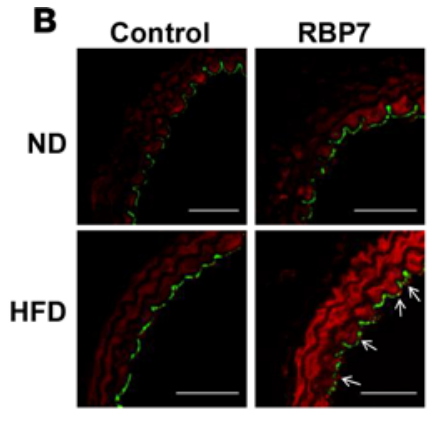

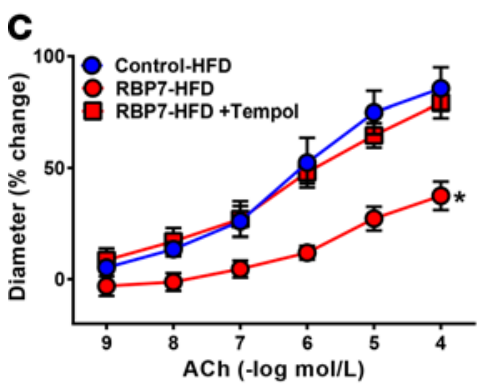
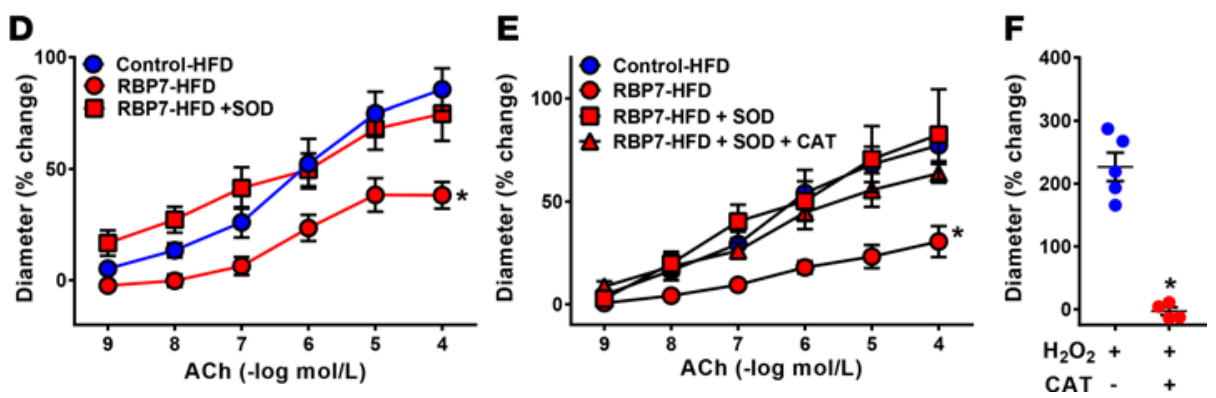

Figure 4. HFD-induced oxidative stress. (A and B) Representative photomicrographs of dihydroethidium (DHE, A) fluorescence and nitrotyrosine immunofluorescence (B) staining of carotid arteries from ND-fed and HFD-fed control and RBP7-deficient mice. White arrows indicate nitrotyrosinepositive endothelial cells that costain with CD31. Scale bars: $200 \mu \mathrm{m}(\mathbf{A})$ and $50 \mu \mathrm{m}$ (B). Quantification of independent replicates is shown. ${ }^{*} P<0.05$ RBP7 HFD vs. RBP7 ND; ${ }^{P} P<0.05$ RBP7 vs. control by 2-way ANOVA; $n=6-8$ per group in $\mathbf{A} ; n=4-5$ per group in $\mathbf{B}$. (C-E) Vasodilation of the basilar artery from control and RBP7-deficient mice fed HFD for 8 weeks was measured in response to acetylcholine (ACh). To measure the response to antioxidant treatment, basilar arteries from RBP7-deficient mice were pretreated with Tempol (C, $1 \mathrm{mmol} / \mathrm{l})$, PEG-SOD (D, $100 \mathrm{U} / \mathrm{ml}$ ), and a combination of PEG-SOD and PEG-catalase (CAT) $(E, 600 \mathrm{U} / \mathrm{ml})$ for 30 minutes in the vessel bath. ${ }^{*} P<0.05$ vs. all other curves by 2-way repeated measures ANOVA; $n=3-8$ per group. $(\mathbf{F})$ Vasodilation of the basilar artery from control mice was measured in response to $\mathrm{H}_{2} \mathrm{O}_{2}$ in vessels preincubated with vehicle or CAT. ${ }^{*} P<0.05$ by Student's $t$ test; $n=5$ per group. All data are the mean \pm SEM. HFD, high-fat diet; ND, normal diet; RBP7, retinol-binding protein 7 .

Adipo $Q$ is an RBP7-dependent PPAR target gene in endothelium. To assess the functional relationship between PPAR $\gamma$ and RBP7, we performed RNA-Seq of RNA isolated from carotid artery from control and RBP7-deficient mice that were incubated in vitro with either DMSO (vehicle) or rosiglitazone. Expression of Adipo $Q$ mRNA was detected in carotid artery samples that were fully cleaned of perivascular adipose tissue, suggesting $A$ dipo $Q$ expression in nonadipocytes in the vessel. The absence of adipocyte contamination in the vessel preparations used for the RNA-Seq analysis was evidenced by the absence of expression of several adipocyte markers including Fgf21, Epsti1, Tcf21, Cited1, and Cd137 (37). According to the RNA-Seq, Adipo $Q$ expression was induced by rosiglitazone 4.7-fold in carotid artery from control mice, but only 1.4-fold in carotid artery from RBP7-deficient mice.

To confirm the RNA-Seq finding, we isolated carotid artery from control and RBP7-deficient mice, incubated a portion of each vessel in either vehicle or rosiglitazone, and then measured expression of AdipoQ and Fabp4 (classically known as aP2) mRNA, a canonical PPAR $\gamma$ target gene, by quantitative real-time reverse transcription PCR (qRT-PCR) comparing the level of induction to the matched vehicle sample. As above, we validated the absence of contaminating adipocytes by measuring expression of several adipocyte-specific markers by qRT-PCR. There was no evidence for the expression of HoxC9, Fgf21, Cited1, and CD137 in carotid artery from control or RBP7-deficient mice incubated with either vehicle or rosiglitazone. Threshold cycle $(\mathrm{Ct})$ values were greater than 35 or undetectable in carotid artery, whereas they were 25-30 in either inguinal or perivascular adipose tissue. Adipo $Q$ was significantly increased in carotid artery from rosiglitazone-treated control mice, but not in rosiglitazone-treated RBP7-deficient mice (Figure 6A). Similarly, induction of Fabp4 expression by rosiglitazone was blunted in carotid artery from RBP7-deficient mice (Figure 6B). These data suggest that PPAR $\gamma$-mediated induction of both Adipo $Q$ and Fabp4 mRNA is RBP7 dependent. To confirm that the rosiglitazone-induced $A$ dipo $Q$ expression was of endothelial origin, endothelial cells and non-endothelial cells were collected from a separate group of rosiglitazone- or DMSO-treated carotid artery. AdipoQ 

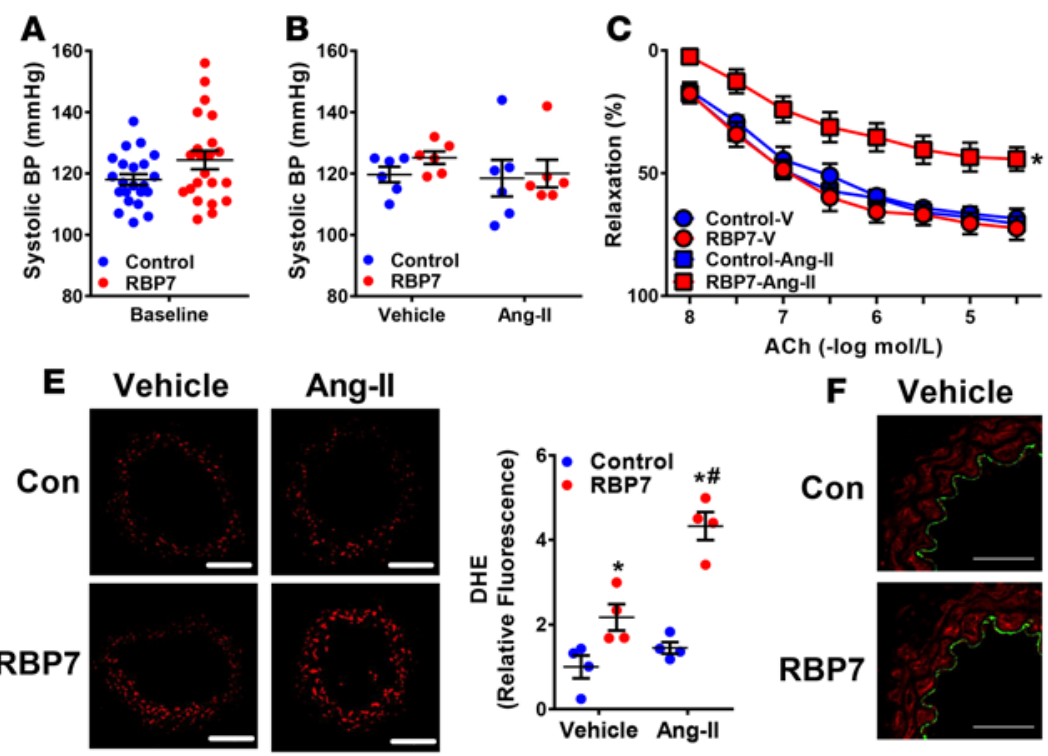

G
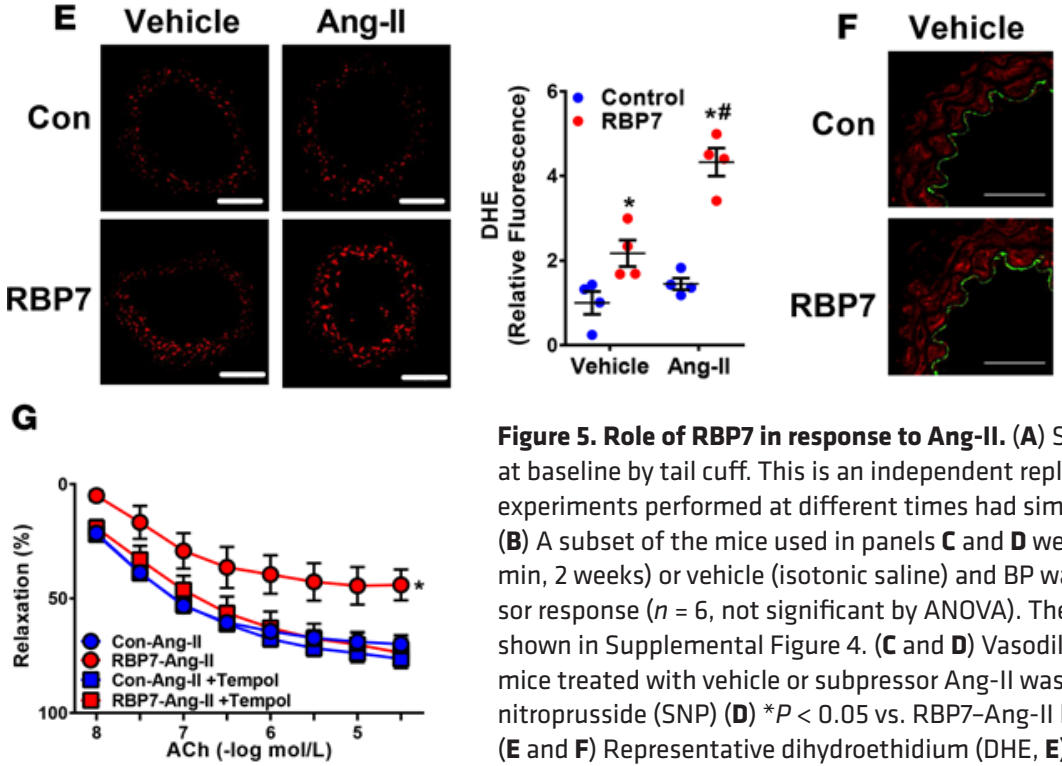
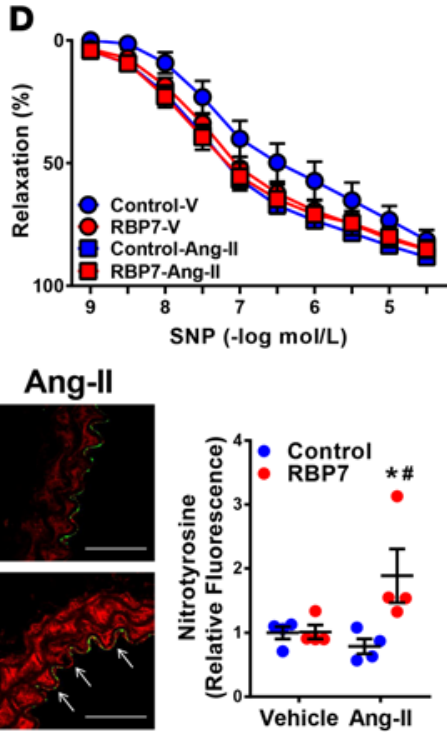

Figure 5. Role of RBP7 in response to Ang-II. (A) Systolic BP was measured in control and RBP7-deficient mice at baseline by tail cuff. This is an independent replication cohort from that shown in Figure 1 to ensure that experiments performed at different times had similar range of BPs ( $n=22$, not significant by Student's $t$ test). (B) A subset of the mice used in panels $\mathbf{C}$ and $\mathbf{D}$ were first treated with a subpressor dose of Ang- 11 (120 ng/kg/ min, 2 weeks) or vehicle (isotonic saline) and BP was measured by tail cuff to confirm the absence of a pressor response ( $n=6$, not significant by ANOVA). The robust hypertensive response to a pressor dose of Ang-II is shown in Supplemental Figure 4. (C and D) Vasodilation of the carotid artery from control and RBP7-deficient mice treated with vehicle or subpressor Ang-II was measured in response to acetylcholine (ACh) (C) and sodium nitroprusside (SNP) (D) ${ }^{*} P<0.05$ vs. RBP7-Ang-Il by 2-way repeated measures (RM) ANOVA; $n=5-8$ per group. (E and F) Representative dihydroethidium (DHE, E) fluorescence and nitrotyrosine immunofluorescence (F) staining conducted in carotid arteries from vehicle or Ang-II-infused control or RBP7-deficient mice. White arrows indicate nitrotyrosine-positive endothelial cells that costain with CD31. Scale bars: $100 \mu \mathrm{m}$ (E) and $50 \mu \mathrm{m}$ (F). Quantification of independent replicates is shown. ${ }^{*} P<0.05$ RBP7 vs. control; ${ }^{*} P<0.05$ RBP7-Ang-II vs. RBP7vehicle by 2-way ANOVA; $n=4$ per group. (C) Vasodilation of the carotid artery from control and RBP7-deficient mice infused with subpressor Ang-II for 2 weeks in the presence or absence of Tempol (1 mmol/l, 30 minutes) was measured in response to ACh. ${ }^{*} P<0.05$ vs. all other curves by 2 -way RM ANOVA; $n=5$ per group. All data are the mean $\pm \mathrm{SEM}$. Ang-II, angiotensin II; RBP7, retinol-binding protein 7; V, vehicle; Con, control.

expression was specifically induced in endothelial cells from carotid artery of control mice, but not in non-endothelial cells, and RBP7-deficiency ablated the induction of AdipoQ in endothelial cells (Figure 6C). As before, the fidelity of the MACS-based cell sorting was confirmed by the presence of Nos3 and Cd31 mRNA in endothelial fractions but not in the non-endothelial fractions (Supplemental Figure 7). To assess the localization of AdipoQ in carotid artery (dissected free of perivascular adipose), dual-immunofluorescence staining was conducted with antisera targeting AdipoQ, and the endothelial cell-specific marker CD31. AdipoQ was detected in cells that costained with CD31 in the intima of the vessel, but no staining was detected in the media, indicating that AdipoQ is expressed selectively in the endothelium (Figure 6E). Importantly, the AdipoQ immunofluorescence signal was induced $1.6 \pm 0.1$-fold (AdipoQ/ CD31) in rosiglitazone-treated carotid artery from control but not in rosiglitazone-treated carotid artery from RBP7-deficient mice $(0.9 \pm 0.2$-fold).

To determine if the RBP7 dependency affects responses under another condition where PPAR $\gamma$ is induced in vivo, we examined whether $A$ dipo $Q$ mRNA was induced in vessels cleaned of perivascular adipose from control and RBP7-deficient mice fed HFD (Figure 6D). Like the induction by rosiglitazone, expression of Adipo $Q$ was induced in control but not RBP7-deficient mice. Immunofluorescence verified that this induction occurred selectively in endothelial cells (Figure 6F). These results indicate that PPAR $\gamma$ induced expression of Adipo $Q$ is both endothelium-specific and RBP7-dependent in the blood vessel.

$R B P 7-m e d i a t e d$ endothelial protection from oxidative stress requires Adipo $Q$. Since AdipoQ is a known adipokine, synthesized and secreted from adipose tissue, experiments were performed to examine AdipoQ expression in different adipose depots and plasma in HFD-fed mice. Expression of AdipoQ mRNA was similar in 
A

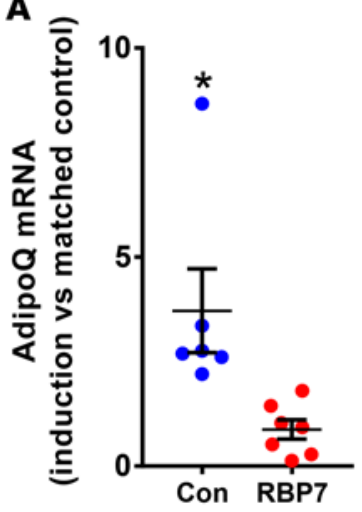

B
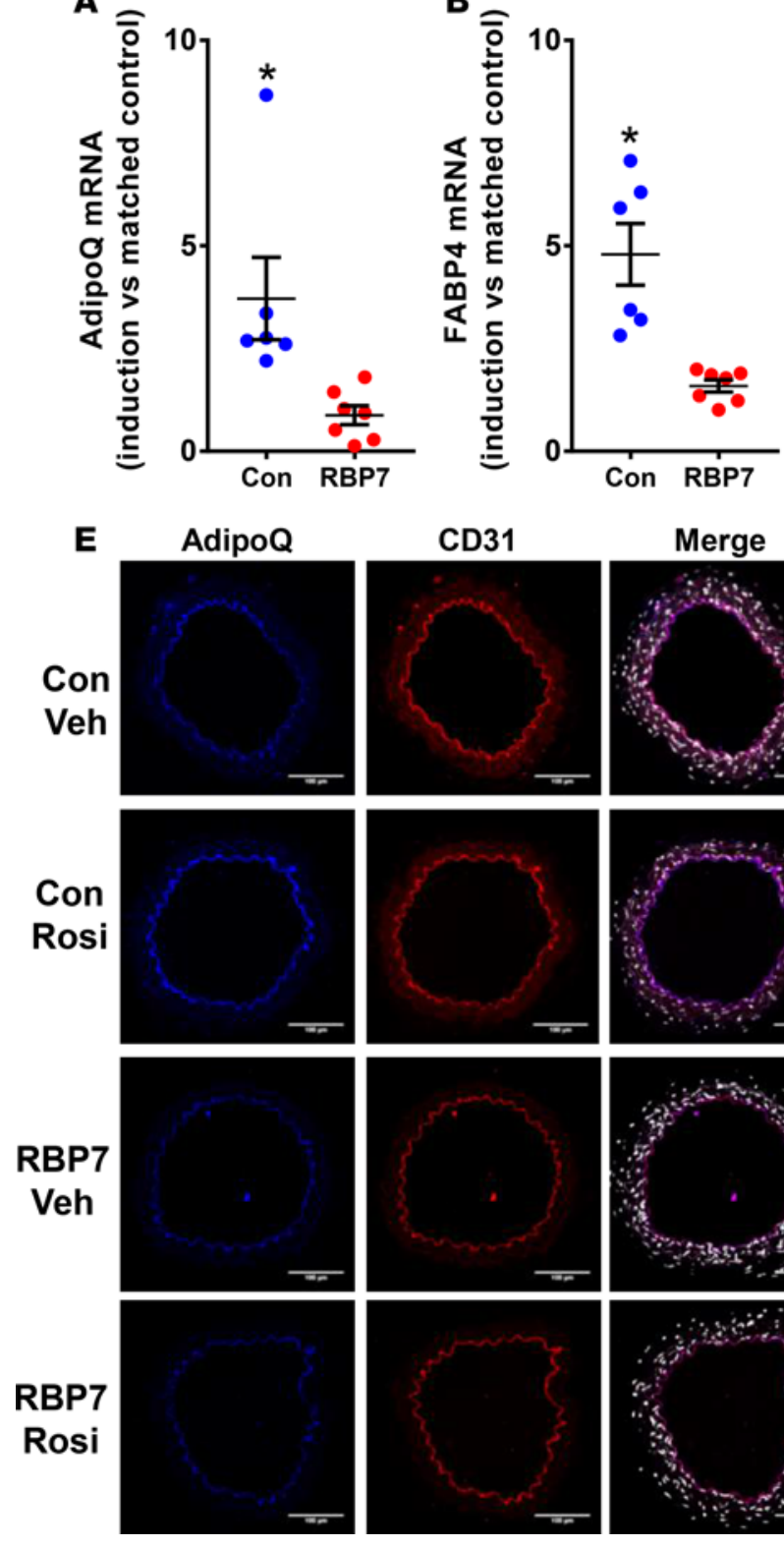

CD31
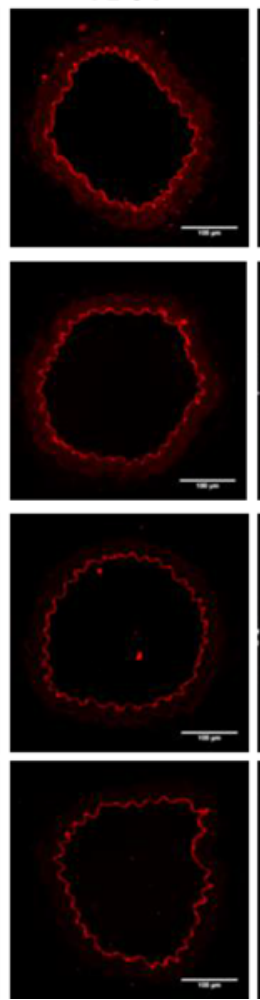

C
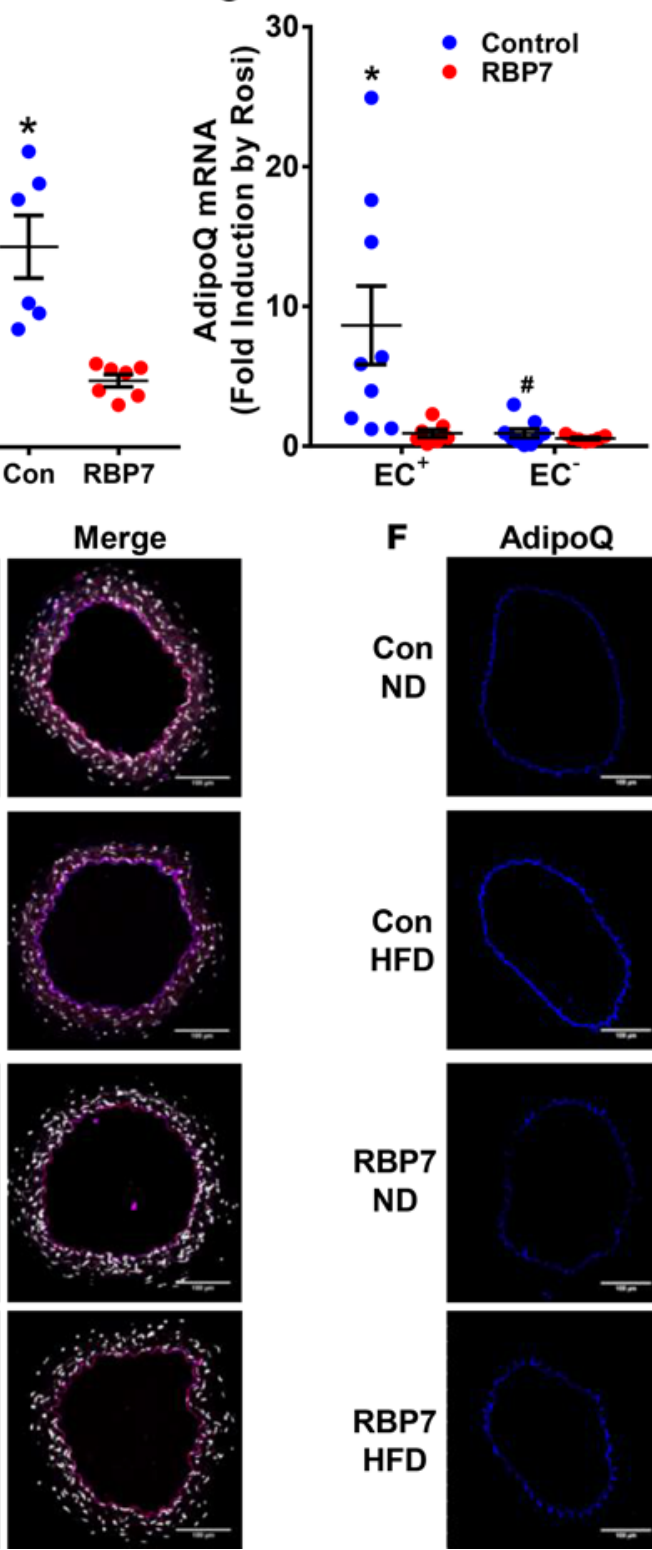

F

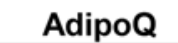

Con

ND
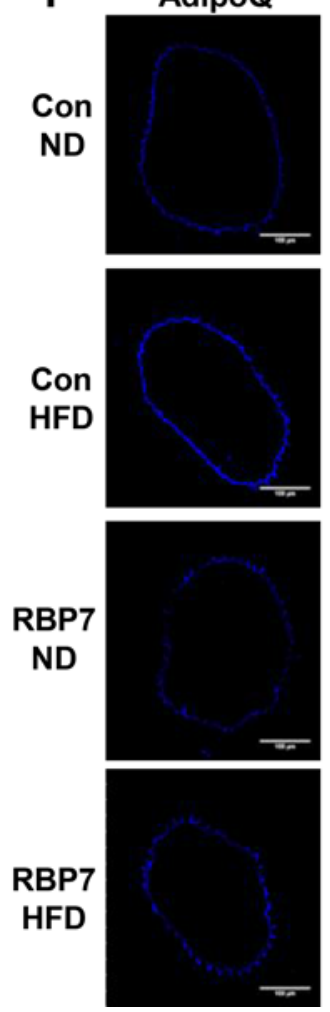
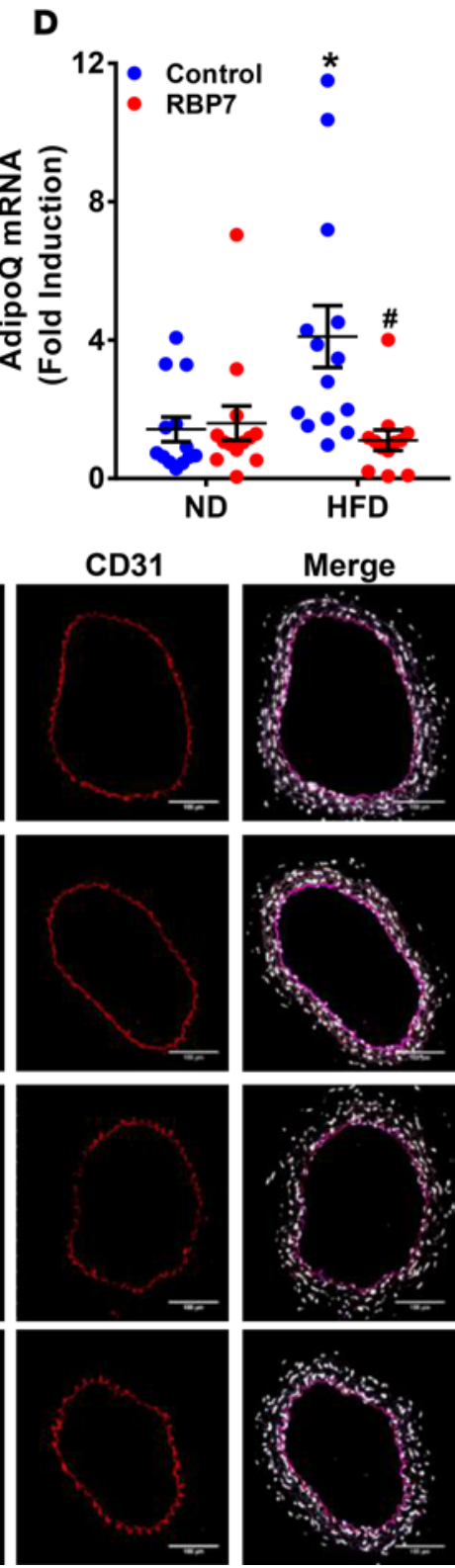

Figure 6. Adiponectin (AdipoQ) is an RBP7-dependent PPAR $\gamma$ target gene. (A and B) Segments of the carotid artery were bisected and matched samples were incubated in vitro with vehicle (DMSO) or rosiglitazone (Rosi) for 24 hours. Quantification of AdipoQ (A) and FABP4 (B) expression was measured by quantitative real-time reverse transcription PCR (qRT-PCR) using CAPDH or $\beta$-actin as the normalization control. The level of induction by Rosi was compared between matched samples. ${ }^{*} P<0.05$ control vs. RBP7-deficient by paired $t$ test; $n=6-7$ per group. (C) Endothelial (EC+) and non-endothelial (EC ${ }^{-}$) cells were magnetically sorted from dissociated single cells isolated from carotid arteries incubated in vitro with vehicle or Rosi for 24 hours. AdipoQ expression was measured by qRT-PCR. ${ }^{*} P<0.05$ control vs. RBP7-deficient; ${ }^{\#} P<0.05$ control EC+ vs. EC- by 2 -way ANOVA; $n=7-9$ per group. (D) AdipoQ mRNA expression was quantified by qRT-PCR from aorta or carotid artery isolated from control or RBP7-deficient mice fed either normal diet (ND) or high-fat diet (HFD) ( $n=12-14$ per group). ${ }^{*} P<$ 0.05 control, HFD vs. ND; ${ }^{*} P<0.05$ HFD, RBP7 vs. control by 2-way ANOVA. Results are the mean \pm SEM. (E and F) Dual immunofluorescence of carotid arteries from control and RBP7-deficient mice treated in vitro with Rosi (10 $\mu \mathrm{M}, 24$ hours) or DMSO (E), or from control and RBP7-deficient mice fed either ND or HFD (F) was performed using antisera detecting AdipoQ (blue) or the endothelium-specific marker CD31 (red). Sections were also stained with DAPI (white/gray). Representative photomicrographs from $n=3-7$ experiments per group are shown. Scale bars: $100 \mu \mathrm{m}$. Con, control; RBP7, retinol-binding protein 7; Veh, vehicle.

perivascular (Supplemental Figure 8A) and inguinal (subcutaneous) adipose tissue (Supplemental Figure 8B), but was modestly decreased in gonadal (visceral) adipose tissue from HFD-fed RBP7-deficient mice (Supplemental Figure 8C). Despite the decrease in gonadal adipose $A d i p o Q$, there was no significant change in the levels of plasma AdipoQ in any group (control ND, $12.4 \pm 1.7$; control HFD, $17.5 \pm 2.0$; RBP7 ND, $13.7 \pm 2.6 ; \mathrm{RBP7} \mathrm{HFD}, 14.2 \pm 2.9 \mu \mathrm{g} / \mathrm{ml} ; n=9-10)$. Thus, changes in vascular function are unlikely the result of a change in circulating AdipoQ. 

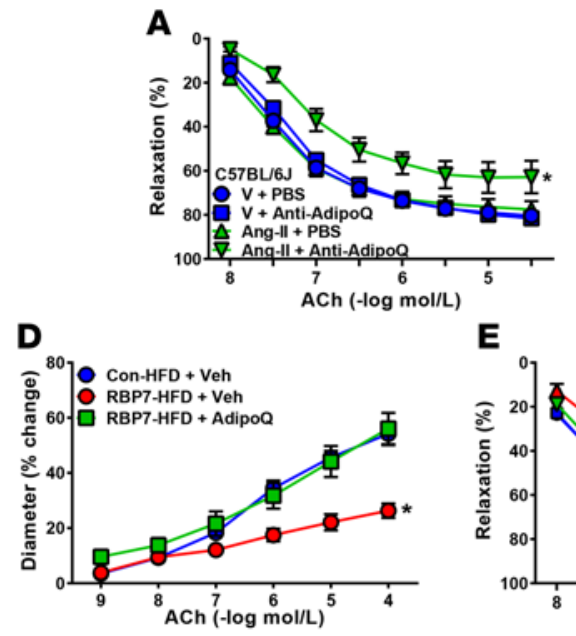

H
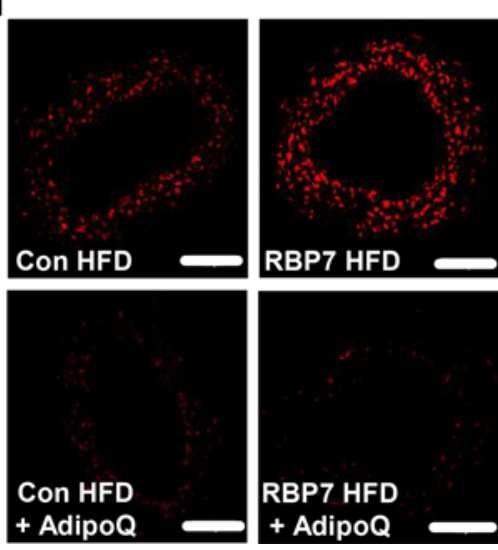

E
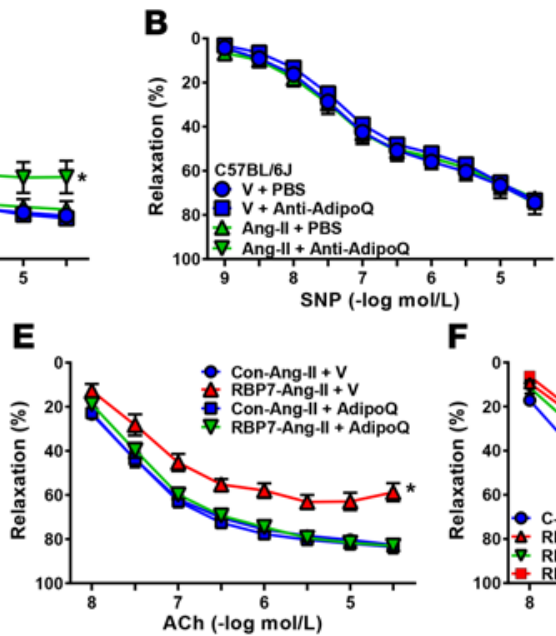

F
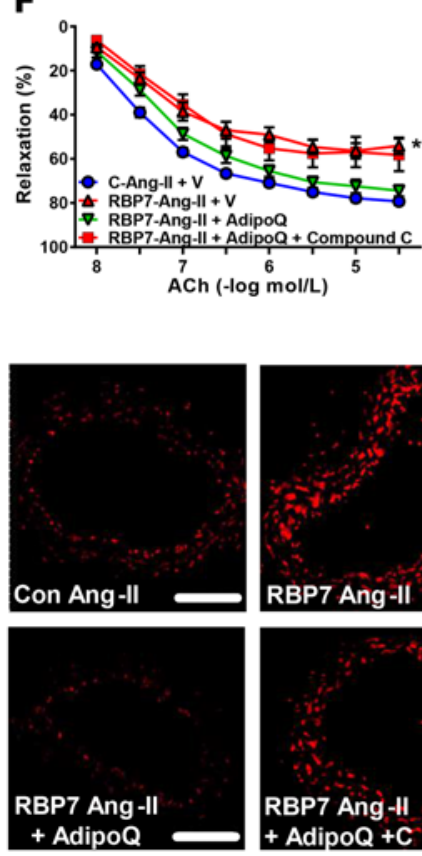

$-P-T^{172}-A M P K \alpha$

-tAMPK $\alpha$

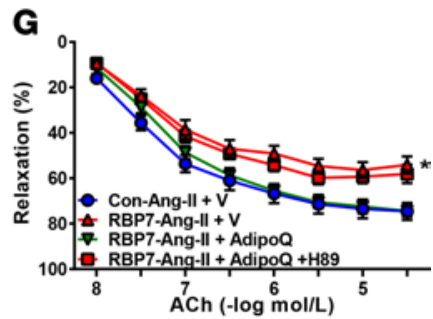

Figure 7. RBP7-mediated antioxidant protection requires adiponectin (AdipoQ). (A and B) Carotid artery from subpressor Ang-II-infused (120 ng/kg/min, 2 weeks) or vehicle-infused (isotonic saline) C57BL/6) mice were first preincubated with anti-AdipoQ antibody (5 $\mu \mathrm{g} / \mathrm{ml})$ or PBS in vitro for $12 \mathrm{hours}$. Vasodilation of carotid arteries was measured in response to acetylcholine (ACh) (A) and sodium nitroprusside (SNP) (B). ${ }^{*} P<0.05$ vs. all other curves by 2 -way repeated measures (RM) ANOVA. $n=6$ per group. (C) Western blot showing phosphorylation of AMPK $\alpha$ at T172 and total AMPK $\alpha$ (tAMPK $\alpha$ ) in mouse lung endothelial cells (MLECs) treated with insulin (In, $100 \mathrm{nM}$ ) and the indicated dose $(\mu \mathrm{g} / \mathrm{ml})$ of AdipoQ. (D) Vasodilation of the basilar artery from HFD-fed RBP7-deficient mice after incubation with recombinant AdipoQ $\left(5 \mu \mathrm{g} / \mathrm{ml}, 4\right.$ hours) in vitro was measured in response to $A C h$. ${ }^{*} P<0.05$ vs. all other curves by 2-way RM ANOVA. $n=5$-8 per group. (E) Vasodilation of the carotid artery from subpressor Ang-II-treated RBP7-deficient and control mice after incubation with recombinant AdipoQ ( $5 \mu \mathrm{g} / \mathrm{ml}, 12$ hours) in vitro was measured in response to ACh. ${ }^{*} P<0.05$ vs. all other curves by 2 -way RM ANOVA. $n=7$ per group. Vasodilation of the carotid artery from subpressor Ang-II-treated RBP7-deficient mice after incubation with recombinant AdipoQ ( $5 \mu \mathrm{g} / \mathrm{ml}, 12 \mathrm{hours})$ in vitro was measured in response to ACh. Vessels were preincubated with the AMPK inhibitor compound C ( $5 \mu \mathrm{mol} / \mathrm{I}, \mathbf{F})$ and PKA inhibitor H89 $(1 \mu \mathrm{mol} / \mathrm{I}, \mathbf{C})$ for 1 hour before AdipoQ. ${ }^{*} P<0.05$ compound C or H89 vs. Ang-II + AdipoQ and control Ang-II by 2-way RM ANOVA. Samples sizes are $n=13$ (control-Ang-II), 9 (RBP7 + Ang-II), 4 (RBP7 + Ang-II + AdipoQ), 6 (RBP7 + Ang-II + AdipoQ + compound C), 7 (RBP7 + Ang-II + AdipoQ + H89). (H and I) Representative photomicrographs of dihydroethidium (DHE) fluorescence staining of carotid arteries from HFD-fed (H) and Ang-II-treated (I) control and RBP7-deficient mice. Scale bars: $100 \mu \mathrm{m}$. Summary data are presented next to each set of micrographs. Results are the mean $\pm \mathrm{SEM}$. ${ }^{*} P<0.05 \mathrm{RBP7}$ vs. control; ${ }^{\#} P<0.05$ AdipoQ vs. vehicle in RBP7-deficient mice; ${ }^{*} P<0.05$ compound C vs. AdipoQ. All analyses were by 2 -way ANOVA. Sample sizes are $n=4$ for the Ang-II experiment and $n=6-7$ for the HFD experiment. Ang-II, angiotensin II; HFD, high-fat diet; RBP7, retinol-binding protein 7; V, vehicle.

Experiments were next performed to examine the mechanistic role of AdipoQ on endothelial function. First, we examined vessels from control C57BL/6J mice infused with vehicle or a subpressor dose of Ang-II. Vessels were then incubated in vitro with anti-AdipoQ antibody or PBS to determine the protective effect of endogenous AdipoQ. Anti-AdipoQ antisera significantly impaired ACh-induced vasodilation in carotid artery from Ang-II-treated, but not from vehicle-treated mice (Figure 7A). There was no difference in the vasodilator response to SNP (Figure 7B). Thus, interference with AdipoQ by anti-AdipoQ antisera phenocopied the loss of RBP7, at least at the level of the endothelial response to ACh. Experiments were next conducted to examine if incubation with exogenous recombinant AdipoQ would restore endothelium-dependent relaxation in HFD-fed or Ang-II-treated RBP7-deficient mice. We confirmed the activity of recombinant AdipoQ by showing that it induced phosphorylation of T172 
A

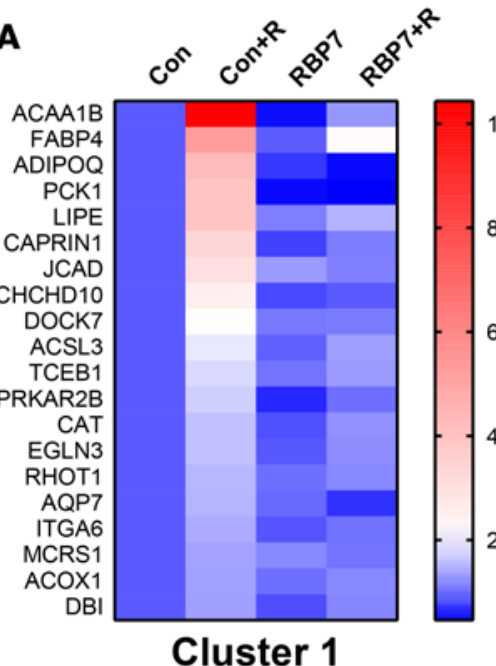

B

Cluster 1

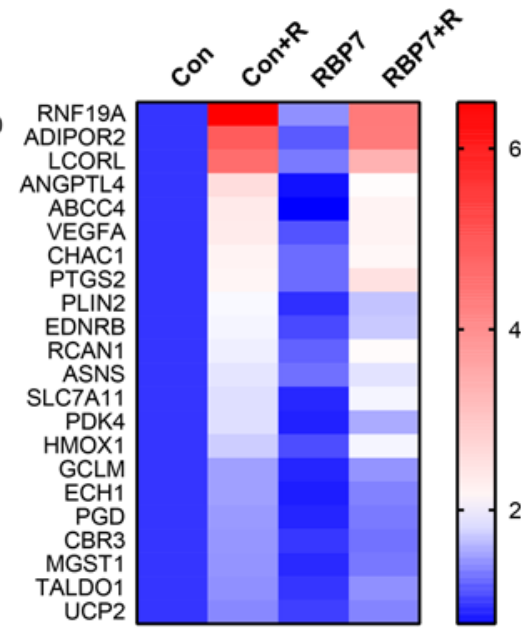

Cluster 2

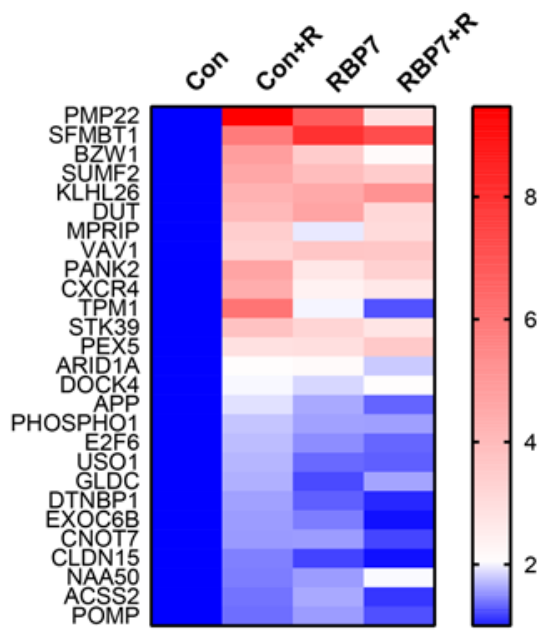

Cluster 3
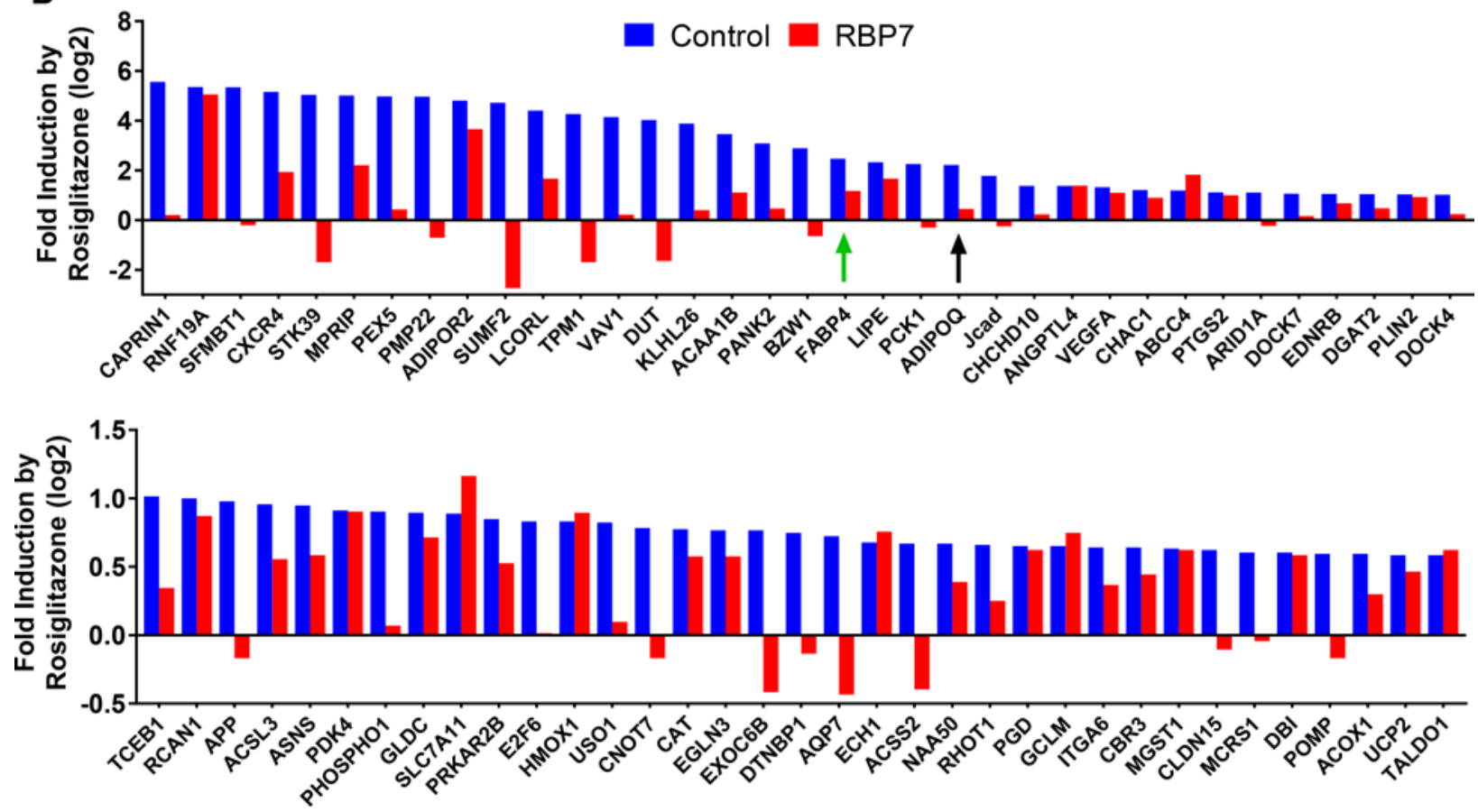

Figure 8. Genome-wide analysis of retinol-binding protein 7 (RBP7) dependency. Carotid artery was isolated from control (Con) and RBP7-deficient mice (RBP7) and incubated in vitro with vehicle or rosiglitazone $(+R)$ for 24 hours $(10 \mu \mathrm{M})$. RNA was isolated and analyzed by RNA-Seq as detailed in the Methods and supplemental methods. (A) Cluster analysis of PPAR $\gamma$ target genes expressed in endothelium of carotid artery. Expression levels (based on number of corrected reads/length of gene/total reads) of each gene were normalized, setting the level in the untreated control to 1.0. The level of expression was pseudocolored using the heatmap feature in GraphPad Prism 7.01. (B) The fold induction by rosiglitazone is compared in control and RBP7-deficient mice. The data were transformed $(\log 2)$ to decrease the scale and to clearly illustrate those samples where rosiglitazone caused a decrease in gene expression. Genes are shown in decreasing order of their induction by rosiglitazone. Green arrow indicates FABP4. Black arrow indicates adiponectin (AdipoQ).

monophosphate-activated protein kinase $\alpha(\mathrm{AMPK} \alpha)$ to the same extent as insulin $(100 \mathrm{nM})$ in MLECs (Figure 7C). AdipoQ completely restored ACh-induced relaxation in basilar artery from HFD-fed RBP7-deficient mice (Figure 7D), and in carotid artery from subpressor Ang-II-infused RBP7-deficient mice (Figure 7E). Coincubation with either compound C (an inhibitor of AMPK) or H89 (an inhibitor of protein kinase A, PKA) ablated the protective effect of AdipoQ on the carotid artery (Figure 7, F and $\mathrm{G})$, consistent with effects being mediated through the AdipoQ receptor (AdipoR) (31). Importantly, there was no difference in expression of AdipoR 1 and AdipoR 2 in carotid artery from control and RBP7deficient mice fed either ND or HFD (Supplemental Figure 9). Mechanistically, AdipoQ incubation reduced superoxide production in response to both HFD (Figure 7H) and subpressor Ang-II (Figure 7I) 


\section{Endothelium}

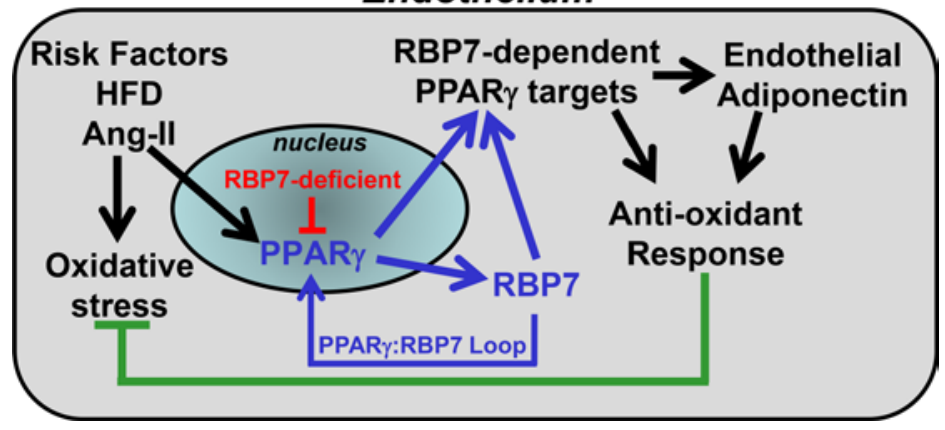

Figure 9. Model Illustrating the PPAR $\gamma /$ RBP7/adiponectin transcriptional hub. PPAR $\gamma$ is a nuclear transcription factor that induces expression of retinol-binding protein 7 (RBP7), an endothelium-specific PPAR $\gamma$ target gene. RBP7 facilitates transcriptional activation of RBP7-dependent PPAR $\gamma$ target genes including adiponectin (AdipoO). Expression of these genes is impaired in the absence of RBP7. Expression of AdipoQ mediates an antioxidant response when the mice are challenged with either high-fat diet (HFD) or angiotensin II (Ang-II). Impairment of this protective response under conditions where either RBP7 or PPAR $\gamma$ is impaired causes oxidative stress and endothelial dysfunction in the presence of cardiovascular stressors.

in RBP7-deficient mice. The protective antioxidant response of AdipoQ to subpressor Ang-II was ablated by compound C (Figure 7I). These data indicate that AdipoQ is a mediator of the protective antioxidant effect of the PPAR $\gamma / \mathrm{RBP} 7$ pathway.

$R B P 7$ is required for induction of some PPAR $\gamma$ target genes. We next analyzed the RNA-Seq data to assess if induction of all PPAR $\gamma$ target genes required RBP7. First, we identified all genes with a statistically significant 1.5-fold (or higher) rosiglitazone-induced increase in gene expression in vessels derived from control mice. Samples with an extremely low level of expression were excluded from the analysis (normalized counts < 0.1). Second, we identified bona fide PPAR $\gamma$ target genes by virtue of being either (a) a known PPAR $\gamma$ target or (b) expressed and having a validated PPAR $\gamma$ binding site in chromatin (ChIP Seq or Chip CHIP) of adipocytes or macrophages $(22,23,38)$. Finally, we identified those genes that are expressed in endothelium on the basis of (a) being expressed in human umbilical vein endothelial cells (39), human aortic endothelial cells, or mouse brain endothelial cells (40); (b) enriched in endothelium versus smooth muscle (comparing expression signatures in GSE3239); or (c) enriched in endothelium by ribosome-affinity purification (25). This bioinformatic enrichment resulted in the identification of 70 genes.

When we normalized expression to the level in the untreated controls, we identified 3 clusters of genes (Figure 8A). The first cluster contains genes that were induced by rosiglitazone in endothelium from control mice but were not induced in RBP7-deficient mice. This cluster included both AdipoQ and Fabp4, and the antioxidant gene Cat. The second cluster of genes was induced by rosiglitazone in endothelium from both control and RBP7-deficient mice, and would be considered RBP7-independent PPAR $\gamma$ target genes. The third cluster of genes was induced in endothelium from control mice and exhibited an increased level of expression in the samples from RBP7-deficient mice. Although the level of expression was increased in RBP7-deficient mice, there was a blunted response to rosiglitazone in the RBP7-deficient samples in cluster 3.

We also examined data by independently comparing the magnitude of rosiglitazone-induced expression in the control samples to the magnitude of rosiglitazone-induced expression in RBP7-deficient samples (Figure 8B). As above, there was a distribution of genes that were RBP7-dependent and RBP7-independent. Interestingly, induction of many PPAR $\gamma$ target genes by rosiglitazone was either ablated or markedly blunted in carotid artery from RBP7-deficient mice, an effect that was particularly notable in those genes exhibiting the most robust induction by rosiglitazone. On the contrary, of the genes induced less than 3-fold, there was roughly an equal number of responses that were RBP7-dependent and RBP7-independent. In sum, these data suggest that there are both RBP7-dependent and RBP7-independent PPAR $\gamma$ target genes expressed in the endothelium. Also, the increased level of baseline expression of some genes (cluster 3) observed in RBP7-deficient mice suggests that there may be some genes where the actions of RBP7 and PPAR $\gamma$ may be in opposition.

\section{Discussion}

The main findings of the present study are that: (a) RBP7 expression is essentially endothelium specific, (b) RBP7 is both a PPAR $\gamma$ target gene itself and an upstream regulator of some PPAR $\gamma$ target genes in the endothelium, (c) PPAR $\gamma$ controls the expression of RBP7-dependent and RBP7-independent genes in the endothelium, (d) RBP7 is required to mediate protection from oxidative stress in response to 2 cardiovascular stressors, HFD and Ang-II, and (e) PPAR $\gamma$ and RBP7 form an antioxidant transcriptional hub that requires the action of AdipoQ, an RBP7-dependent PPAR $\gamma$ target gene in endothelium. Thus, RBP7 essentially acts as a PPAR $\gamma$ cofactor in the endothelium. 
RBP7 was first identified as being expressed in heart, epididymal fat, and skeletal muscle (24), but more recently was identified as an endothelium-enriched transcript (25), and to be selectively expressed in endothelial cells in heart, skeletal muscle, adipose tissues, salivary gland and thymus, particularly in small arteries and arterioles (26). Consistent with this, we show that RBP7 was expressed in endothelial cells in kidney, lung, and aorta, and importantly, the induction of RBP7 mRNA expression by a PPAR $\gamma$ agonist occurred only in endothelial cells of the carotid artery. Loss of $R B P 7$ expression in the endothelium resulted in oxidative stress when the mice were treated with pro-oxidant inducers, such as HFD or Ang-II. However, there was no obvious baseline vascular phenotype in RBP7-deficient mice, suggesting that RBP7 may be dispensable in the absence of a cardiovascular stressor. Indeed, there was no difference in the level of baseline AdipoQ or Fabp 4 mRNA expression in the carotid artery of control compared with RBP7-deficient mice.

Like RBP7-deficiency, interference with PPAR $\gamma$ in the endothelium through targeted expression of a hypertension-causing dominant-negative mutation in $\operatorname{PPAR} \gamma(7)$ has no effect under baseline conditions, but results in oxidative stress and endothelial dysfunction in response to HFD and Ang-II $(12,13)$. This effect of PPAR $\gamma$ interference is particularly prominent in smaller blood vessels such as the basilar artery, which supplies and regulates blood to the brain (41). Other studies reported that deletion of PPAR $\gamma$ in the endothelium results in oxidative stress-dependent endothelial dysfunction either at baseline or in the presence of HFD $(42,43)$. That interference with PPAR $\gamma$ and ablation of RBP7 have the same phenotype is consistent with the notion that both may function in the same physiological pathway in the endothelium.

RBP7 is a member of the large family of FABPs (44). RBP7 can bind all-trans-, 13-cis-, and 9-cis-retinol, precursors for retinyl ester and retinoic acid (24). As a family, retinoids are needed for cardiovascular health. It is also notable that retinoids are ligands for the retinoid X receptor (RXR), which is the obligate heterodimeric partner for PPAR $\gamma$. RBP7 was previously shown to play a role in lipid and energy metabolism with protective effects observed in RBP7-deficient mice fed HFD (20). Although these data would suggest that RBP7 may have some detrimental effects in mice fed HFD, it is notable that in our study we did not observe significant differences in body weight or weight gain, glucose homeostasis, or liver steatosis when control mice fed a HFD were compared with RBP7-deficient mice fed a HFD. Thus, we concluded that the endothelial dysfunction selectively observed in RBP7-deficient mice occurred independently of metabolic changes.

In considering a mechanism for the action of RBP7, it is instructive to examine how other members of the FABP family function. Interestingly, there is evidence that FABPs can modulate the activity of nuclear receptors. The intracellular lipid-binding proteins CRABP-II and FABP5 control the partitioning of retinoic acid between retinoic acid receptor (RAR) and PPAR $\beta \delta$ to activate either a prosurvival or proapoptotic pathway (45). Similarly, 2 different FABPs may enhance activity of PPAR $\gamma$ or PPAR $\beta \delta$ (46). Mechanistically, FABP1 and FABP2 were reported to enhance PPAR $\alpha$ transcriptional activity by translocating from the cytoplasm to the nucleus in a ligand-dependent manner, presumably to deliver ligand to the PPAR $\alpha$ transcriptional complex (47). Therefore, it becomes attractive to hypothesize that RBP7 might regulate the transcriptional activity of certain PPAR $\gamma$ target genes such as Adipo $Q$ in the endothelium by a similar mechanism.

Another prominent member of the FABP family is Fabp4, a canonical PPAR $\gamma$ target gene that is also expressed in the endothelium. We demonstrated that induction of Fabp4 expression by rosiglitazone in the carotid artery was also RBP7 dependent. Previous studies suggest that high levels of plasma FABP4 are associated with endothelial dysfunction in patients with type 2 diabetes, which may be due in part to impaired insulin-mediated NO signaling in endothelium $(48,49)$. It is uncertain if FABP4 serves a function similar to that of RBP7 in the endothelium, whether they carry similar ligands, or if they control distinct sets of PPAR $\gamma$ response genes. Indeed, an important unanswered question in nuclear receptor biology is how nuclear receptors determine which genes to activate. This is a particularly important question when one considers that there may be as many as 5,000 binding sites for PPAR $\gamma$ in the genome.

We identified that endothelial AdipoQ is an RBP7-dependent PPAR $\gamma$ target gene. AdipoQ is a protective adipokine secreted from adipose tissue. Deletion of AdipoQ causes impaired endothelium-dependent relaxation by enhancing oxidative stress and reducing NO bioavailability $(32,50)$, whereas AdipoQ supplementation improved endothelial dysfunction in diabetic mice by suppressing oxidative stress (51). AdipoQ has also been reported to be expressed in kidney (52) and endothelium (33). Consistent with this, we identified that AdipoQ was selectively expressed in the endothelium of carotid artery, its expression was induced by rosiglitazone and HFD, and deletion of RBP7 markedly blunted this effect. Moreover, by measuring expression of adipocyte-specific genes, we showed that our vessel preparations were free of contaminating perivascular 
adipocytes. That blocking endogenous AdipoQ accelerated endothelial dysfunction in response to subpressor Ang-II in normal mice suggests that endothelial AdipoQ is functionally important. Similarly, oxidative stress and endothelial dysfunction were rescued by the addition of full-length recombinant mouse AdipoQ to carotid and basilar arteries. Furthermore, we demonstrated that AdipoQ improves endothelial function through AMPK- and PKA-dependent pathways, consistent with previous evidence that these pathways are involved in the vascular beneficial effects of AdipoQ $(31,53)$. The finding that there was no difference in the level of circulating, perivascular, or subcutaneous adipose tissue AdipoQ in HFD-fed control and RBP7deficient mice lends further support to the hypothesis that AdipoQ generated directly in the endothelium is an important component of RBP7-mediated antioxidant protection.

In conclusion, our data suggest that PPAR $\gamma$ and RBP7 form a transcriptional regulatory circuit (or hub) in the endothelium that controls the oxidative state of the vessel (Figure 9). This is achieved by activating expression of AdipoQ under conditions where the antioxidant state is threatened. This regulatory circuit may not be necessary under baseline conditions either because other antioxidant mechanisms predominate or baseline expression or circulating levels of AdipoQ is sufficient to maintain an antioxidant state. On the contrary, impairment of this regulatory circuit either by mutation of PPAR $\gamma$ or loss of RBP7 prevents the induction of AdipoQ, resulting in oxidative stress and endothelial dysfunction. Prolonged endothelial dysfunction in the presence of other cardiovascular risk factors may then predispose to hypertension or atherosclerosis. Thus, the PPAR $\gamma / \mathrm{RBP} 7 /$ AdipoQ axis could be a novel therapeutic intervention for treating endothelial dysfunction in cardiovascular disease.

\section{Methods}

Experimental procedures not described here can be found in the supplemental material.

Animals and diets. Male and female RBP7-deficient mice and their littermate controls were used in this study. Male C57BL/6J mice (Jackson Laboratory) aged 8-12 weeks were utilized in the anti-AdipoQ experiments. Mice were fed with a HFD for 8 or 20 weeks (rodent diet with $45 \% \mathrm{kcal} \%$ fat, D12451, Research Diets Inc.), or standard chow diet as control (Harlan/Teklad Mouse/Rat Irradiated Diet 7913), beginning at 4 weeks of age. All mice were housed under a 12-hour light/dark cycle at a controlled temperature $\left(23^{\circ} \mathrm{C}\right)$, with water and food ad libitum.

Ex vivo studies of vascular reactivity. Established techniques for measurement of basilar and carotid artery function have been described previously $(12,13,54)$. Briefly, after mice were euthanized with pentobarbital (50 mg/mouse i.p.), brain and carotid arteries were quickly removed and placed in oxygenated Krebs buffer (in mmol/1: $118.3 \mathrm{NaCl}, 4.7 \mathrm{KCl}, 1.2 \mathrm{MgSO}_{4}, 1.2 \mathrm{KH}_{2} \mathrm{PO}_{4}, 25 \mathrm{NaHCO}_{3}, 2.5 \mathrm{CaCl}_{2}$ and $11 \mathrm{D}$-glucose). Basilar arteries were isolated and cannulated onto glass micropipettes filled with oxygenated Krebs buffer in an organ chamber, and transferred to a pressurized myograph system (DMT, 110P) with equilibration for 30 minutes at $60 \mathrm{mmHg}$ under no-flow conditions before protocols were initiated. For carotid arteries, both left and right carotid arteries were utilized with the loose tissue of adventitia layer rapidly removed in oxygenated Krebs buffer. Vascular rings (3-4 mm in length) were suspended in an organ bath and connected to force transducers to measure isometric tension. The rings were allowed to equilibrate for 45 minutes at a resting tension of $0.25 \mathrm{~g}$ before evaluating their reactivity. For relaxation responses, both basilar and carotid arteries were preconstricted with the thromboxane A2 mimetic U46619. In some experiments, arteries were preincubated with Tempol (1 mmol/1), PEG-SOD (100 U/ml), or PEG-CAT (600 U/ml) for 30 minutes.

For some experiments, basilar artery and carotid artery rings, prepared as described above, were incubated in Dulbecco's Modified Eagle Medium/Nutrient Mixture F-12 (Gibco) supplemented with $1 \%$ penicillin-streptomycin at $37^{\circ} \mathrm{C}$ in an atmosphere of $95 \%$ air and $5 \% \mathrm{CO}_{2}$. Mouse full-length AdipoQ $(5 \mu \mathrm{g} / \mathrm{ml})$ and anti-AdipoQ $(5 \mu \mathrm{g} / \mathrm{ml})$ were added in the absence or presence of AMPK inhibitor compound C (5 $\mu \mathrm{mol} / 1)$ or PKA inhibitor H89 (1 $\mu \mathrm{mol} / 1)$ (31). After incubation (basilar artery, 4 hours; carotid artery, 12-13 hours), arteries were transferred to oxygenated Krebs buffer, and mounted to a pressure or wire myograph to evaluate vascular function.

Immunofluorescence staining. A double-immunofluorescence (AdipoQ and CD31; nitrotyrosine and CD31) method was performed as previously reported $(33,36)$. Carotid arteries with adventitia and blood removed were fixed in $4 \%$ paraformaldehyde overnight at $4{ }^{\circ} \mathrm{C}$, following immersion in $30 \%$ sucrose until the sample dropped to the bottom of a vial. Samples were then embedded in OCT compound and kept at $-80^{\circ} \mathrm{C}$ until sectioning. Sections $(10 \mu \mathrm{m}$ thick) were cut using a cryostat, incubated with goat anti-AdipoQ $(15 \mu \mathrm{g} / \mathrm{ml}, \mathrm{R} \& \mathrm{D}$ Systems Inc., AF1119) and rat anti-CD31 (1:50, BD Biosciences, 553370) in PBS containing 1\% normal don- 
key serum. Donkey anti-goat IgG-conjugated Alexa 647 (Life Technologies, A-21447) and goat anti-rat IgGconjugated Alexa 568 (Life Technologies, A-11077) were utilized as the secondary antibodies. To determine the level and localization of nitrotyrosine, sections were incubated with rabbit anti-nitrotyrosine (1:50, EMD Millipore, 06-284) and rat anti-CD31 as described above in PBS containing 5\% goat serum. Goat anti-rabbit IgG-conjugated Alexa 568 (Life Technologies, A-11036) and goat anti-rat IgG-conjugated Alexa 488 (Life Technologies, A-11006) were used as the secondary antibodies. Cell nuclei were counterstained with DAPI (Vector Laboratories). Images were visualized using confocal microscopy (Zeiss LSM710) and analyzed using ImageJ software (NIH).

RNA-Seq. RNA-Seq was conducted on RNA purified from carotid arteries from control and RBP7deficient mice after they were incubated in vitro with rosiglitazone (10 $\mu \mathrm{M}, n=3-4$ per group). The perivascular adipose tissue, adventitia layer, and blood were carefully removed under ice-cold Krebs buffer, and snap-frozen in liquid nitrogen. RNA was generated immediately after snap freezing using the Trizol method. RNA-Seq protocols were conducted at the Iowa Institute of Human Genetics, Genomics Division. Paired-end reads (100-bp) from the Illumina HiSeq 2500 were aligned to the mouse transcriptome (UCSC known genes, build mm10) with the Kallisto program. The quality of the reads was assessed using the FastQC program (version 0.11.2). For all samples, more than $98 \%$ of the reads were of high quality (phred score $>20$ ). Differential expression was assessed using the Kallisto companion program Sleuth. The gene list was then filtered by $P$ value $(<0.05)$, fold change $(\geq 1.5)$, and baseline level of expression (average transcripts per million or TPM $\geq 0.1$ ). Data from the RNA-Seq experiment have been deposited at the NCBI's Gene Expression Omnibus database (GEO GSE88706).

Statistics. Results represent means \pm SEM. Significance was analyzed using 1-, 2-, or 3-way ANOVA (repeated measures when appropriate) followed by Bonferroni post hoc tests, or Student's $t$ test where required (Systat Software or Graphpad Prism). $P$ less than 0.05 was considered a statistically significant difference. Specific tests are denoted in the figure legends.

Study approval. Care and use of mice in this study met the standard guidelines for the care and use of experimental animals by the NIH. All animal procedures were approved by the University of Iowa IACUC.

\section{Author contributions}

$\mathrm{CH}$ conceived and designed the research, performed experiments, analyzed data, interpreted results, and drafted and revised the manuscript. HK analyzed RNA-Seq results and revised the manuscript. KL performed some qRT-PCR experiments. XL designed some experiments and performed RNA isolation for some experiments. DRD performed radiotelemetry-implanted surgery. JW performed MACS cell sorting. SCI performed Western blot experiments in MLECs. SV developed the RBP7-deficient mice and provided conceptual input throughout the study. FWQ conceived and designed the research, interpreted results, and edited and revised the manuscript. CDS conceived and designed the research, analyzed data, interpreted results, edited and revised the manuscript, and approved the final version of the manuscript.

\section{Acknowledgments}

We thank Brandon S. Davies for providing us with MLECs. This work was supported through research grants from the NIH (HL084207, HL125603, HL131689), and American Heart Association to CDS (15SFRN23480000). The authors gratefully acknowledge the research support of the Roy J. Carver Trust.

Address correspondence to: Curt D. Sigmund, Roy J. Carver Chair in Hypertension Research, Chair and Department Executive Officer, Department of Pharmacology, Director, UI Center for Hypertension Research, Roy J. and Lucille A. Carver College of Medicine University of Iowa, Iowa City, Iowa 52242, USA. Phone: 319.335.7410; E-mail: curt-sigmund@uiowa.edu.

1. Cai H, Harrison DG. Endothelial dysfunction in cardiovascular diseases: the role of oxidant stress. Circ Res. 2000;87(10):840-844.

2. Barak Y, et al. PPAR gamma is required for placental, cardiac, and adipose tissue development. Mol Cell. 1999;4(4):585-595.

3. He W, et al. Adipose-specific peroxisome proliferator-activated receptor gamma knockout causes insulin resistance in fat and liver but not in muscle. Proc Natl Acad Sci USA. 2003;100(26):15712-15717.

4. Hevener AL, et al. Muscle-specific Pparg deletion causes insulin resistance. Nat Med. 2003;9(12):1491-1497.

5. Lehmann JM, Moore LB, Smith-Oliver TA, Wilkison WO, Willson TM, Kliewer SA. An antidiabetic thiazolidinedione is a high affinity ligand for peroxisome proliferator-activated receptor gamma (PPAR gamma). J Biol Chem. 1995;270(22):12953-12956. 
6. Dormandy JA, et al. Secondary prevention of macrovascular events in patients with type 2 diabetes in the PROactive Study (PROspective pioglitAzone Clinical Trial In macroVascular Events): a randomised controlled trial. Lancet. 2005;366(9493):1279-1289.

7. Barroso I, et al. Dominant negative mutations in human PPARgamma associated with severe insulin resistance, diabetes mellitus and hypertension. Nature. 1999;402(6764):880-883.

8. Savage DB, et al. Human metabolic syndrome resulting from dominant-negative mutations in the nuclear receptor peroxisome proliferator-activated receptor-gamma. Diabetes. 2003;52(4):910-917.

9. Halabi CM, et al. Interference with PPAR gamma function in smooth muscle causes vascular dysfunction and hypertension. Cell Metab. 2008;7(3):215-226.

10. Pelham CJ, et al. Cullin-3 regulates vascular smooth muscle function and arterial blood pressure via PPAR $\gamma$ and RhoA/Rhokinase. Cell Metab. 2012;16(4):462-472.

11. Agbor LN, et al. Cullin-3 mutation causes arterial stiffness and hypertension through a vascular smooth muscle mechanism. JCI Insight. 2016;1(19):e91015.

12. Beyer AM, et al. Endothelium-specific interference with peroxisome proliferator activated receptor gamma causes cerebral vascular dysfunction in response to a high-fat diet. Circ Res. 2008;103(6):654-661.

13. Hu C, Lu KT, Mukohda M, Davis DR, Faraci FM, Sigmund CD. Interference with PPAR $\gamma$ in endothelium accelerates angiotensin II-induced endothelial dysfunction. Physiol Genomics. 2016;48(2):124-134.

14. Nicol CJ, Adachi M, Akiyama TE, Gonzalez FJ. PPARgamma in endothelial cells influences high fat diet-induced hypertension. Am J Hypertens. 2005;18(4 Pt 1):549-556.

15. Calnek DS, Mazzella L, Roser S, Roman J, Hart CM. Peroxisome proliferator-activated receptor gamma ligands increase release of nitric oxide from endothelial cells. Arterioscler Thromb Vasc Biol. 2003;23(1):52-57.

16. Delerive $P$, et al. Peroxisome proliferator-activated receptor activators inhibit thrombin-induced endothelin- 1 production in human vascular endothelial cells by inhibiting the activator protein-1 signaling pathway. Circ Res. 1999;85(5):394-402.

17. Mukohda M, Stump M, Ketsawatsomkron P, Hu C, Quelle FW, Sigmund CD. Endothelial PPAR- $\gamma$ provides vascular protection from IL-1ß-induced oxidative stress. Am J Physiol Heart Circ Physiol. 2016;310(1):H39-H48.

18. Sigmund CD. Endothelial and vascular muscle PPARgamma in arterial pressure regulation: lessons from genetic interference and deficiency. Hypertension. 2010;55(2):437-444.

19. Marx N, Bourcier T, Sukhova GK, Libby P, Plutzky J. PPARgamma activation in human endothelial cells increases plasminogen activator inhibitor type-1 expression: PPARgamma as a potential mediator in vascular disease. Arterioscler Thromb Vasc Biol. 1999;19(3):546-551.

20. Zizola CF, Schwartz GJ, Vogel S. Cellular retinol-binding protein type III is a PPARgamma target gene and plays a role in lipid metabolism. Am J Physiol Endocrinol Metab. 2008;295(6):E1358-E1368.

21. Keen HL, et al. Bioinformatic analysis of gene sets regulated by ligand-activated and dominant-negative peroxisome proliferator-activated receptor gamma in mouse aorta. Arterioscler Thromb Vasc Biol. 2010;30(3):518-525.

22. Nielsen R, et al. Genome-wide profiling of PPARgamma:RXR and RNA polymerase II occupancy reveals temporal activation of distinct metabolic pathways and changes in RXR dimer composition during adipogenesis. Genes Dev. 2008;22(21):2953-2967.

23. Lefterova MI, et al. PPARgamma and C/EBP factors orchestrate adipocyte biology via adjacent binding on a genome-wide scale. Genes Dev. 2008;22(21):2941-2952.

24. Vogel S, et al. Characterization of a new member of the fatty acid-binding protein family that binds all-trans-retinol. J Biol Chem 2001;276(2):1353-1360.

25. Zhou P, et al. Interrogating translational efficiency and lineage-specific transcriptomes using ribosome affinity purification. Proc Natl Acad Sci USA. 2013;110(38):15395-15400.

26. Caprioli A, Zhu H, Sato TN. CRBP-III:lacZ expression pattern reveals a novel heterogeneity of vascular endothelial cells. Genesis. 2004;40(3):139-145.

27. Antoniades C, Antonopoulos AS, Tousoulis D, Stefanadis C. Adiponectin: from obesity to cardiovascular disease. Obes Rev. 2009;10(3):269-279.

28. Kadowaki T, Yamauchi T, Kubota N, Hara K, Ueki K, Tobe K. Adiponectin and adiponectin receptors in insulin resistance, diabetes, and the metabolic syndrome. J Clin Invest. 2006;116(7):1784-1792.

29. Devaraj S, Torok N, Dasu MR, Samols D, Jialal I. Adiponectin decreases C-reactive protein synthesis and secretion from endothelial cells: evidence for an adipose tissue-vascular loop. Arterioscler Thromb Vasc Biol. 2008;28(7):1368-1374.

30. Ouchi N, et al. Association of hypoadiponectinemia with impaired vasoreactivity. Hypertension. 2003;42(3):231-234.

31. Wong WT, et al. Adiponectin is required for $\operatorname{PPAR} \gamma$-mediated improvement of endothelial function in diabetic mice. Cell Metab. 2011;14(1):104-115.

32. Tan KC, et al. Hypoadiponectinemia is associated with impaired endothelium-dependent vasodilation. J Clin Endocrinol Metab. 2004;89(2):765-769.

33. Komura N, et al. Adiponectin protein exists in aortic endothelial cells. PLoS ONE. 2013;8(8):e71271.

34. Moraes-Vieira PM, Yore MM, Dwyer PM, Syed I, Aryal P, Kahn BB. RBP4 activates antigen-presenting cells, leading to adipose tissue inflammation and systemic insulin resistance. Cell Metab. 2014;19(3):512-526.

35. Brouwers O, et al. Hyperglycaemia-induced impairment of endothelium-dependent vasorelaxation in rat mesenteric arteries is mediated by intracellular methylglyoxal levels in a pathway dependent on oxidative stress. Diabetologia. 2010;53(5):989-1000.

36. Hilgers RH, Xing D, Gong K, Chen YF, Chatham JC, Oparil S. Acute O-GlcNAcylation prevents inflammation-induced vascular dysfunction. Am J Physiol Heart Circ Physiol. 2012;303(5):H513-H522.

37. de Jong JM, Larsson O, Cannon B, Nedergaard J. A stringent validation of mouse adipose tissue identity markers. Am J Physiol Endocrinol Metab. 2015;308(12):E1085-E1105.

38. Lefterova MI, et al. Cell-specific determinants of peroxisome proliferator-activated receptor gamma function in adipocytes and macrophages. Mol Cell Biol. 2010;30(9):2078-2089.

39. Hart T, Komori HK, LaMere S, Podshivalova K, Salomon DR. Finding the active genes in deep RNA-seq gene expression studies. 
BMC Genomics. 2013;14:778.

40. Zhang Y, et al. An RNA-sequencing transcriptome and splicing database of glia, neurons, and vascular cells of the cerebral cortex. J Neurosci. 2014;34(36):11929-11947.

41. De Silva TM, et al. Role of peroxisome proliferator-activated receptor- $\gamma$ in vascular muscle in the cerebral circulation. Hypertension. 2014;64(5):1088-1093.

42. Kleinhenz JM, et al. Disruption of endothelial peroxisome proliferator-activated receptor-gamma reduces vascular nitric oxide production. Am J Physiol Heart Circ Physiol. 2009;297(5):H1647-H1654.

43. Kanda T, et al. PPARgamma in the endothelium regulates metabolic responses to high-fat diet in mice. J Clin Invest. 2009;119(1):110-124.

44. Chmurzyńska A. The multigene family of fatty acid-binding proteins (FABPs): function, structure and polymorphism. $J$ Appl Genet. 2006;47(1):39-48.

45. Schug TT, Berry DC, Shaw NS, Travis SN, Noy N. Opposing effects of retinoic acid on cell growth result from alternate activation of two different nuclear receptors. Cell. 2007;129(4):723-733.

46. Tan NS, et al. Selective cooperation between fatty acid binding proteins and peroxisome proliferator-activated receptors in regulating transcription. Mol Cell Biol. 2002;22(14):5114-5127.

47. Hughes ML, et al. Fatty acid-binding proteins 1 and 2 differentially modulate the activation of peroxisome proliferator-activated receptor $\alpha$ in a ligand-selective manner. J Biol Chem. 2015;290(22):13895-13906.

48. Aragonès G, et al. Fatty acid-binding protein 4 is associated with endothelial dysfunction in patients with type 2 diabetes. Atherosclerosis. 2010;213(1):329-331.

49. Aragonès G, Saavedra P, Heras M, Cabré A, Girona J, Masana L. Fatty acid-binding protein 4 impairs the insulin-dependent nitric oxide pathway in vascular endothelial cells. Cardiovasc Diabetol. 2012;11:72.

50. Cao Y, et al. Endothelial dysfunction in adiponectin deficiency and its mechanisms involved. J Mol Cell Cardiol. 2009;46(3):413-419.

51. Lee S, Zhang H, Chen J, Dellsperger KC, Hill MA, Zhang C. Adiponectin abates diabetes-induced endothelial dysfunction by suppressing oxidative stress, adhesion molecules, and inflammation in type 2 diabetic mice. Am J Physiol Heart Circ Physiol. 2012;303(1):H106-H115.

52. Perri A, et al. Adiponectin is expressed and secreted by renal tubular epithelial cells. J Nephrol. 2013;26(6):1049-1054.

53. Ouedraogo R, et al. Adiponectin suppression of high-glucose-induced reactive oxygen species in vascular endothelial cells: evidence for involvement of a cAMP signaling pathway. Diabetes. 2006;55(6):1840-1846.

54. Faraci FM, Modrick ML, Lynch CM, Didion LA, Fegan PE, Didion SP. Selective cerebral vascular dysfunction in Mn-SODdeficient mice. J Appl Physiol. 2006;100(6):2089-2093. 